\title{
Statistical estimates for channel flows driven by a pressure gradient
}

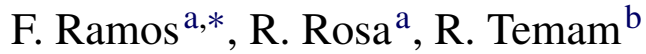 \\ ${ }^{a}$ Instituto de Matemática, Universidade Federal do Rio de Janeiro, Caixa Postal 68530 Ilha do Fundão, Rio de Janeiro, RJ 21945-970, Brazil \\ ${ }^{\mathrm{b}}$ Department of Mathematics, Indiana University, Bloomington, IN 47405, USA
}

Available online 20 March 2008

\begin{abstract}
We present rigorous estimates for some physical quantities related to turbulent and non-turbulent channel flows driven by a uniform pressure gradient. Such results are based on the concept of stationary statistical solutions, which is related to the notion of ensemble averages for flows in statistical equilibrium. We provide a lower bound estimate for the mean skin friction coefficient and improve on a previous upper bound estimate for the same quantity; both estimates are derived in terms of the Reynolds number. We also present lower and upper bound estimates for the mean rate of energy dissipation, the mean longitudinal bulk velocity (in the direction of the pressure gradient), and the mean kinetic energy in terms of various physical parameters. In particular, we obtain an upper bound related to the energy dissipation law, namely that the mean rate of energy dissipation is essentially bounded by a non-dimensional universal constant times the cube of the mean longitudinal bulk velocity over a characteristic macro-scale length. Finally, we investigate the scale-by-scale energy injection due to the pressure gradient, proving an upper bound estimate for the decrease of this energy injection as the scale length decreases.
\end{abstract}

(c) 2008 Elsevier B.V. All rights reserved.

Keywords: Navier-Stokes equations; Turbulence; Skin friction coefficient; Energy dissipation rate; Kolmogorov energy dissipation law; Channel flows

\section{Introduction}

While the existence of exact solutions of the Navier-Stokes equations are not available in general, most of the classical research on turbulence theory consists of approximate methods based on a few exact deductions, supplemented with intuitive hypotheses about the nature of the phenomenon, such as scaling assumptions and moment truncation models; see for example [1,24].

Recently, part of the theoretical research on turbulence has been concentrated on deriving rigorous bounds on characteristic quantities of turbulent flows directly from the equations of motion. These results are important to substantiate the ones obtained via the classical approximation methods.

Decomposing the turbulent flow into a stationary background flow and a fluctuation component, and using variational methods, Constantin and Doering derived rigorous results for the long-time averaged rate of energy dissipation of flows in some geometries, in particular for the channel flow driven by a pressure gradient, in which case the estimate also yields an estimate for the mean skin friction coefficient; see [3,4].

Meanwhile, rigorous results were recently established for the three-dimensional theory of homogeneous stationary statistical turbulence in $[8,12-14]$ using the concepts of stationary statistical solutions of the Navier-Stokes equations and generalized time average measures, and using energy-type methods.

This paper presents a combination of those results in the specific case of channel flows driven by a uniform pressure gradient. More specifically, we extend the upper bound estimate for the long-time averaged skin friction coefficient, obtained in [4], to general

\footnotetext{
* Corresponding address: Department of Computer Science and Applied Mathematics, Weizmann Institute of Science. Rehovot 76100 , Israel. Tel.: +972 8934 4279; fax: +972 89346023 .

E-mail addresses: fabio.ramos@weizmann.ac.il (F. Ramos), rrosa@ufrj.br (R. Rosa), temam@indiana.edu (R. Temam).
} 
stationary statistical solutions, slightly simplifying their proof and slightly improving their estimates. We also obtain a lower bound estimate for the skin friction coefficient, which cannot be obtained by the variational principle method of [4]. More precisely, we show that for every stationary statistical solution and for high-Reynolds-number flows, the corresponding skin friction coefficient $C_{f}$ satisfies

$$
\frac{12}{R e} \leq C_{f} \leq 0.484+O\left(\frac{1}{R e}\right)
$$

where the Reynolds number is defined by $R e=h U / \nu$, with $h$ being the height of the channel and $U$, the mean longitudinal bulk velocity.

The lower-bound estimate for $C_{f}$ is optimal in the sense that the stationary statistical solution is arbitrary and may be concentrated on the plane Poiseuille flow (which exists in a mathematical sense but is unstable for high-Reynolds-number flows), for which $C_{f}=12 / R e$. The upper bound is of the same order as that obtained in [4] but it yields a nearly $19 \%$ improvement on the leading order constant term (from 0.597 to 0.484) over the estimate obtained in [4]. However, the upper-bound estimate might not be optimal since heuristic arguments and flow experiments suggest that $C_{f} \sim(\ln \mathrm{Re})^{-2}$ for high-Reynolds-number turbulent flows.

We also give upper and lower bound estimates for some other physical quantities, such as the mean energy dissipation rate, the mean kinetic energy, and the mean longitudinal bulk velocity. In particular, we prove an upper bound estimate related to the energy dissipation law, namely that for high-Reynolds-number flows the mean rate of energy dissipation is essentially bounded by a non-dimensional universal constant times the cube of the mean longitudinal bulk velocity $U$ over the height $h$ of the channel:

$$
\epsilon \leq\left(0.054+O\left(\frac{1}{R e^{2}}\right)\right) \frac{U^{3}}{h} .
$$

Notice that the leading order constant term is much lower than unity, and as pointed out in [3], it shows that this result is substantially more than a formalized classical dimensional analysis argument.

Finally, we study the scale-by-scale energy injection term due to the pressure gradient. We show that the energy injected per unit time into the modes larger than or equal to $\kappa$ is bounded by a term proportional to $\kappa^{-3 / 2}$. The motivation for the study of the decrease of energy injection rate comes from the Kolmogorov theory of turbulence. This theory argues that for turbulent flows there is a certain range of scales much lower than the energy injection scales and greater than the energy dissipative scales in which the kinetic energy is transferred to the small scales at a nearly constant rate equal to the energy dissipation rate. This theory was proposed in the idealized case of locally homogeneous turbulence, away from the boundaries, under the assumption that the energy injection is concentrated on the large scales. However, it is known from experiments that for wall bounded turbulence this hypothesis needs to be corrected $[4,24,18]$. In particular, the energy injection occurs at arbitrarily small scales. The estimates presented in this article yield an upper bound on the rate of decrease of energy injection rate as the scale length decreases.

Part of the motivation for this work was to compare the variational method (e.g. [3,4]) with the energy method (e.g. [10-14]). We found that in the particular case of the channel flow, the variational method gives the best upper bound estimate for the skin friction coefficient but cannot be applied to yield a lower bound, while the energy method gives both bounds, with the lower bound estimate being sharp in a suitable sense but with the upper bound being unrealistic.

The remaining part of this paper is organized as follows. In the next section we introduce the convenient mathematical setting used throughout the work. In Section 3, we recall the definition of stationary statistical solutions of the Navier-Stokes equations and present some related results. In Section 4, we rigorously define the characteristic quantities that will be estimated, such as the mean energy dissipation rate, mean kinetic energy, mean longitudinal bulk velocity, and mean skin friction coefficient. In Section 5, we establish a relation between stationary statistical solutions and time averages. In Sections 6 and 7, we explicitly derive rigorous bounds for the aforementioned physical quantities, utilizing both methods of [12-14] and of [3,4]. In Section 8, we list the explicit values of the aforementioned physical quantities for the specific case of laminar Poiseuille flow, verifying that some of the results obtained in Sections 6 and 7 are optimal in some sense. In Section 9, we conclude the work with a discussion about the scale-byscale energy injection.

\section{Mathematical framework of the Navier-Stokes equations}

We consider an incompressible Newtonian flow confined to a rectangular periodic channel and driven by a uniform pressure gradient. More precisely, the velocity vector field $\mathbf{u}=\left(u_{1}, u_{2}, u_{3}\right)$ of the fluid satisfies the incompressible Navier-Stokes equations

$$
\frac{\partial \mathbf{u}}{\partial t}-v \Delta \mathbf{u}+(\mathbf{u} \cdot \nabla) \mathbf{u}+\nabla p=\frac{P}{L_{x}} \mathbf{e}_{1}, \quad \nabla \cdot \mathbf{u}=0,
$$

in the domain $\Omega=\left(0, L_{x}\right) \times\left(0, L_{y}\right) \times(0, h)$. The scalar $p$ is the kinematic pressure. We denote by $\mathbf{x}=(x, y, z)$ the space variable. The boundary conditions are no-slip on the planes $z=0$ and $z=h$ and periodic in the $x$ and $y$ directions, with periods $L_{x}$ and $L_{y}$, 
respectively, for both $\mathbf{u}$ and $p$. The parameter $P / L_{x}$ denotes the magnitude of the applied pressure gradient. The parameter $v>0$ is the kinematic viscosity, $\mathbf{e}_{1}$ is the unit vector in the $x$ direction, and $L_{x}, L_{y}, h, P>0$. We sometimes refer to the direction $x$ of the pressure gradient as the longitudinal direction.

The mathematical formulation of the Navier-Stokes equations in this geometry can be easily adapted from the no-slip or fullyperiodic case developed in [5,12,17,21,23].

The formulation yields a functional equation for the time-dependent velocity field $\mathbf{u}=\mathbf{u}(t)$ of the form:

$$
\frac{\mathrm{d} \mathbf{u}}{\mathrm{d} t}=\mathbf{F}(\mathbf{u})=\mathbf{f}_{P}-v A \mathbf{u}-B(\mathbf{u}, \mathbf{u}),
$$

where

$$
\mathbf{f}_{P}=\frac{P}{L_{x}} \mathbf{e}_{1}
$$

Two fundamental spaces are defined by

$$
H=\left\{\mathbf{u}=\left.\mathbf{w}\right|_{\Omega} ; \begin{array}{l}
\mathbf{w} \in\left(L_{\text {loc }}^{2}\left(\mathbb{R}^{2} \times(0, h)\right)\right)^{3}, \quad \nabla \cdot \mathbf{w}=0, \\
\mathbf{w}\left(x, y+L_{x}, y, z\right)=\mathbf{w}(x, y, z), \\
\mathbf{w}_{3}(x, y, 0)=\mathbf{w}(x, y, z), \quad \text { a.e. }(x, y, z) \in \mathbf{w}_{3}(x, y, h)=0, \quad \text { a.e. }(x, y) \in \mathbb{R}^{2} .
\end{array}\right\} .
$$

and

$$
V=\left\{\mathbf{u}=\left.\mathbf{w}\right|_{\Omega} ; \begin{array}{l}
\mathbf{w} \in\left(H_{\mathrm{loc}}^{1}\left(\mathbb{R}^{2} \times(0, h)\right)\right)^{3}, \quad \nabla \cdot \mathbf{w}=0, \\
\mathbf{w}\left(x, y+L_{x}, y, z\right)=\mathbf{w}(x, y, z), \\
\mathbf{w}(x, y, 0)=\mathbf{w}(x, y, h)=0, \quad \text { a.e. }(x, y) \in \mathbb{R}^{2} .
\end{array}\right\} .
$$

The inner products in $H$ and $V$ are denoted respectively by

$$
(\mathbf{u}, \mathbf{v})=\int_{\Omega} \mathbf{u}(\mathbf{x}) \cdot \mathbf{v}(\mathbf{x}) \mathrm{d} \mathbf{x}, \quad((\mathbf{u}, \mathbf{v}))=\int_{\Omega} \sum_{i=1,3} \frac{\partial \mathbf{u}}{\partial x_{i}} \cdot \frac{\partial \mathbf{v}}{\partial x_{i}} \mathrm{~d} \mathbf{x},
$$

and the associated norms by $|\mathbf{u}|_{0}=(\mathbf{u}, \mathbf{u})^{1 / 2},\|\mathbf{u}\|=((\mathbf{u}, \mathbf{u}))^{1 / 2}$.

We identify $H$ with its dual and consider the dual space $V^{\prime}$ of $V$, so that $V \subseteq H \subseteq V^{\prime}$, with the injections being continuous and each space dense in the following one. We also denote by $H_{\mathrm{w}}$ the space $H$ endowed with its weak topology.

We denote by $P_{\mathrm{LH}}$ the (Leray-Helmhotz) orthogonal projector in $L^{2}(\Omega)^{3}$ onto the subspace $H$. The operator $A$ in $(2)$ is the Stokes operator given by $A \mathbf{u}=-P_{\mathrm{LH}} \Delta \mathbf{u}$. The term $B(\mathbf{u}, \mathbf{v})=P_{\mathrm{LH}}((\mathbf{u} \cdot \nabla) \mathbf{v})$ is a bilinear term associated with the inertial term. Moreover, since the Stokes operator is a positive self-adjoint operator on $H$, we consider its powers $A^{s}, s \in \mathbb{R}$, with domain $D\left(A^{s}\right)$. We have $V=D\left(A^{1 / 2}\right)$ and its dual $V^{\prime}=D\left(A^{-1 / 2}\right)$.

The Stokes operator possesses a complete orthonormal basis of eigenvectors in $H,\left\{\mathbf{w}_{j, l, k}\right\}_{j, l, k}$, of the form

$$
\mathbf{w}_{j, l, k}(x, y, z)=\exp \left(\mathrm{i} \pi\left(\frac{j x}{L_{x}}+\frac{l y}{L_{y}}\right)\right) \hat{\mathbf{w}}_{j, l, k}(z),
$$

where $(j, l, k) \in \mathbb{Z} \times \mathbb{Z} \times \mathbb{N}, A \mathbf{w}_{j, l, k}=\lambda_{j, l, k} \mathbf{w}_{j, l, k}$, and each $\hat{\mathbf{w}}_{j, l, k}(z)$ is a suitably normalized eigenfunction of a one-dimensional eigenvalue problem, with $0<\lambda_{j, l, k} \rightarrow \infty$, when $j, l, k \rightarrow \infty$. We write the spectral expansion of $\mathbf{u}$ in this basis as

$$
\mathbf{u}(x, y, z)=\sum_{j, l, k} \hat{u}_{j, l, k} \mathbf{w}_{j, l, k}(x, y, z), \quad \hat{u}_{j, l, k}=\left(\mathbf{u}, \mathbf{w}_{j, l, k}\right) .
$$

To each eigenvalue $\lambda_{j, l, k}$ we associate a wavenumber $\kappa=\kappa_{j, l, k}=\lambda_{j, l, k}^{1 / 2}$. Since $\mathbf{u} \in V$ vanishes on the top and bottom walls, Poincaré inequality applies, yielding a bound on $|\mathbf{u}|_{0}$ in terms of $\|\mathbf{u}\|$. In fact, we have precisely

$$
|\mathbf{u}|_{0}^{2} \leq \lambda_{1}^{-1}\|\mathbf{u}\|^{2}
$$

where $\lambda_{1}=\pi^{2} / h^{2}$ is the smallest positive eigenvalue of the Stokes operator on this geometry. The smallest positive wavenumber is $\kappa_{1}=\lambda_{1}^{1 / 2}=\pi / h$.

We define the component $\mathbf{u}_{\kappa}$ of the vector field $\mathbf{u}$, for a single wavenumber $\kappa$, by

$$
\mathbf{u}_{\kappa}=\sum_{\kappa, l, k=\kappa} \hat{u}_{j, l, k} \mathbf{w}_{j, l, k},
$$


and the component $\mathbf{u}_{\kappa^{\prime}, \kappa^{\prime \prime}}$ with a range of wavenumbers $\left[\kappa^{\prime}, \kappa^{\prime \prime}\right)$ by

$$
\mathbf{u}_{\kappa^{\prime}, \kappa^{\prime \prime}}=\sum_{\kappa^{\prime} \leq \kappa<\kappa^{\prime \prime}} \mathbf{u}_{\kappa} .
$$

We then write the Navier-Stokes equations projected on those components in the form

$$
\frac{\mathrm{d} \mathbf{u}_{\kappa^{\prime}, \kappa^{\prime \prime}}}{\mathrm{d} t}+v A \mathbf{u}_{\kappa^{\prime}, \kappa^{\prime \prime}}+B(\mathbf{u}, \mathbf{u})_{\kappa^{\prime}, \kappa^{\prime \prime}}\left(\mathbf{f}_{P}\right)_{\kappa^{\prime}, \kappa^{\prime \prime}} .
$$

Taking the inner product in $H$ of the bilinear term with a third variable yields a trilinear term

$$
b(\mathbf{u}, \mathbf{v}, \mathbf{w})=(B(\mathbf{u}, \mathbf{v}), \mathbf{w}),
$$

which is defined for $\mathbf{u}, \mathbf{v}, \mathbf{w}$ in $V$. An important relation for the trilinear term is the orthogonality property

$$
b(\mathbf{u}, \mathbf{v}, \mathbf{v})=0,
$$

for $\mathbf{u}, \mathbf{v} \in V$. From this relation comes the following anti-symmetry property

$$
b(\mathbf{u}, \mathbf{v}, \mathbf{w})=-b(\mathbf{u}, \mathbf{w}, \mathbf{v})
$$

for $\mathbf{u}, \mathbf{v}, \mathbf{w} \in V$.

\section{Statistical solutions and the Reynolds equations}

A mathematical framework for the conventional theory of turbulence is based on the concept of stationary statistical solutions of the Navier-Stokes equations. This amounts to considering the space $H$ as a probability space with the $\sigma$-algebra of the Borel sets of $H$ and endowed with a Borel probability measure. The ensemble averages are then regarded as averages with respect to this Borel probability measure. In the three-dimensional case we work mostly with the weak topology. Fortunately the Borel $\sigma$-algebra generated by the weakly open sets coincides with that for the open sets in the strong topology. Since $H$ is a separable Hilbert space every Borel probability measure is automatically regular (see for instance [20]) in the sense that the measure of a measurable set $E$ can be approximated from below by the measure of compact sets included in $E$ and from above by the measure of open sets containing $E$. An important consequence of the regularity of a Borel probability measure is the density of the continuous functions (or just weakly continuous functions) in the space of integrable functions.

We say that a measure $\mu$ in $H$ is carried by a measurable set $E$ if $E$ has full measure in $H$, i.e. $\mu(H \backslash E)=0$. The support of a Borel probability measure $\mu$ is the smallest closed set which carries $\mu$. The ensemble averages are regarded as averages with respect to a Borel probability measure $\mu$ on $H$. If $\varphi: H \rightarrow \mathbb{R}$ is a Borel function representing some physical information $\varphi$ (u) extracted from a velocity field $\mathbf{u}$, such as kinetic energy, velocity, enstrophy, etc., then its mean value is

$$
\langle\varphi\rangle=\int_{H} \varphi(\mathbf{u}) \mathrm{d} \mu(\mathbf{u}) .
$$

The reader is referred to [12] for more details.

Now, we define a class of Borel functions that are particularly useful for a rigorous definition of stationary statistical solutions of the Navier-Stokes equations.

Definition 3.1. We define the class $\mathcal{T}$ of test functions to be the set of real-valued functionals $\Psi=\Psi(\mathbf{u})$ on $H$ that are bounded on bounded subsets of $H$ and such that the following conditions hold:

(1) For any $\mathbf{u} \in V$, the Fréchet derivative $\Psi^{\prime}(\mathbf{u})$ taken in $H$ along $V$ exists. More precisely, for each $\mathbf{u} \in V$, there exists an element in $H$, denoted $\Psi^{\prime}(\mathbf{u})$, such that

$$
\frac{\left|\Psi(\mathbf{u}+\mathbf{v})-\Psi(\mathbf{u})-\left(\Psi^{\prime}(\mathbf{u}), \mathbf{v}\right)\right|_{0}}{|\mathbf{v}|_{0}} \rightarrow 0 \quad \text { as }|\mathbf{v}|_{0} \rightarrow 0, \mathbf{v} \in V .
$$

(2) $\Psi^{\prime}(\mathbf{u}) \in V$ for all $\mathbf{u} \in V$, and $\mathbf{u} \rightarrow \Psi^{\prime}(\mathbf{u})$ is continuous and bounded as a function from $V$ into $V$.

For example, we can take the cylindrical test functions $\Psi: H \rightarrow \mathbb{R}$ of the form $\Psi(\mathbf{u})=\psi\left(\left(\mathbf{u}, \mathbf{g}_{1}\right), \ldots,\left(\mathbf{u}, \mathbf{g}_{m}\right)\right)$, where $\psi$ is a $C^{1}$ scalar function on $\mathbb{R}^{m}, m \in \mathbb{N}$, with compact support, and $\mathbf{g}_{1}, \ldots, \mathbf{g}_{m}$ belong to $V$. In this case we have

$$
\Psi^{\prime}(\mathbf{u})=\sum_{j=1}^{m} \partial_{j} \psi\left(\left(\mathbf{u}, \mathbf{g}_{j}\right), \ldots,\left(\mathbf{u}, \mathbf{g}_{j}\right)\right) \mathbf{g}_{j},
$$


where $\partial_{j} \psi$ denotes the derivative of $\psi$ with respect to the $j$-th variable. It follows that $\Psi^{\prime}(\mathbf{u}) \in V$ since it is a linear combination of the $\mathbf{g}_{j}$, and $\Psi^{\prime}$ is bounded in $V$ since $\psi$ has compact support.

Now, we recall the definition of stationary statistical solutions of the Navier-Stokes equations.

Definition 3.2. A stationary statistical solution of the Navier-Stokes equation is a Borel probability measure $\mu$ on $H$ such that

(1) $\int_{H}\|\mathbf{u}\|^{2} \mathrm{~d} \mu(\mathbf{u})<\infty$;

(2) $\int_{H}\left(\mathbf{F}(\mathbf{u}), \Psi^{\prime}(\mathbf{u})\right) \mathrm{d} \mu(\mathbf{u})=0$, for any $\Psi \in \mathcal{T}$, where $\mathbf{F}(\mathbf{u})$ is as in (2);

(3) $\int_{e_{1} \leq|\mathbf{u}|_{0}^{2} / 2<e_{2}}\left\{v\|\mathbf{u}\|^{2}-\left(\mathbf{f}_{P}, \mathbf{u}\right)\right\} \mathrm{d} \mu(\mathbf{u}) \leq 0$, for all $0 \leq e_{1}<e_{2} \leq+\infty$.

The first condition means that an arbitrary stationary statistical solution has finite mean enstrophy. This is natural when compared with individual solutions, whose time averages have bounded enstrophy uniformly with respect to the time interval. It also guarantees that the $V^{\prime}$ norm of $\mathbf{F}(\mathbf{u})=\mathbf{f}-v A \mathbf{u}-B(\mathbf{u}, \mathbf{u})$ is $\mu$-integrable, so that condition (2) makes sense for $\Psi^{\prime}$ continuous and bounded in $V$, as required in the definition of test function $\Psi \in \mathcal{T}$.

The last condition in the definition above is an energy-type inequality, and one can deduce from it that the support of a stationary statistical solution is included in the weak attractor $\mathcal{A}_{w}$, see [12,15], which is bounded in $H$ according to

$$
\begin{aligned}
|\mathbf{u}|_{0} & \leq \frac{\nu G^{*}}{\kappa_{1}^{1 / 2}}=\frac{v h^{1 / 2}}{\pi^{1 / 2}} G^{*}, \quad \forall \mathbf{u} \in \mathcal{A}_{w}, \\
G^{*} & =\frac{h^{1 / 2}}{v^{2} \pi^{1 / 2}}\left|A^{-1 / 2} \mathbf{f}_{P}\right|_{0},
\end{aligned}
$$

where $G^{*}$ is a nondimensional number called the Grashof number.

The concept of a stationary statistical solution is regarded as a generalization of the notion of an invariant measure for the case in which a semigroup may not be well-defined, as in the three-dimensional Navier-Stokes equations. Note in particular that the definition of stationary statistical solutions makes no reference to the solution operator. And in the two-dimensional case, in which a semigroup is well-defined, both the notions of stationary statistical solutions and of invariant measures turn out to be equivalent (see e.g. $[6,7,12])$.

Due to the above regularity properties of stationary statistical solutions (finite mean enstrophy and support bounded in $H$ ), the mean value $\langle\varphi(\mathbf{u})\rangle$ can be defined not only for weakly continuous functions bounded in $H$ but for any real-valued function $\varphi$ which is continuous in $V$ and satisfies the estimate

$$
|\varphi(\mathbf{u})|_{0} \leq C\left(|\mathbf{u}|_{0}\right)\left(1+v^{-2} \lambda_{1}^{1 / 2}\|\mathbf{u}\|^{2}\right), \quad \forall \mathbf{u} \in V
$$

where $C\left(|\mathbf{u}|_{0}\right)$ is bounded on bounded subsets of $H$. Important examples of such $\varphi$ are $|\mathbf{u}|_{0}^{2}$, $\|\mathbf{u}\|^{2}$, and, as we shall see, $b\left(\mathbf{u}_{\kappa_{1}, \kappa}, \mathbf{u}_{\kappa_{1}, \kappa}, \mathbf{u}_{\kappa, \infty}\right)$, and $b\left(\mathbf{u}_{\kappa, \infty}, \mathbf{u}_{\kappa, \infty}, \mathbf{u}_{\kappa_{1}, \kappa}\right)$.

By a duality argument we can extend the ensemble averages to functions with values in some function spaces. More precisely, we define the mean velocity field $\langle\mathbf{u}\rangle$ and the mean value $\langle B(\mathbf{u}, \mathbf{u})\rangle$ of the inertial term by

$$
\begin{aligned}
& (\langle\mathbf{u}\rangle, \mathbf{v})=\int_{H}(\mathbf{u}, \mathbf{v}) \mathrm{d} \mu(\mathbf{u}), \quad \forall \mathbf{v} \in V^{\prime}, \\
& (\langle B(\mathbf{u}, \mathbf{u})\rangle, \mathbf{v})=\int_{H}(B(\mathbf{u}, \mathbf{u}), \mathbf{v}) \mathrm{d} \mu(\mathbf{u}), \quad \forall \mathbf{v} \in D\left(A^{3 / 8}\right) .
\end{aligned}
$$

The mean flow $\langle\mathbf{u}\rangle$ is a vector field on $\Omega$ with $\langle\mathbf{u}\rangle \in V$, while $\langle B(\mathbf{u}, \mathbf{u})\rangle \in D\left(A^{-3 / 8}\right)$.

Since we assume statistical equilibrium, the stationary form of the Reynolds equations can be recovered within this framework; see also [19]:

Proposition 3.1. Given a stationary statistical solution in the sense of Definition 3.2, the following functional form of the Reynolds equations hold in $V^{\prime}$ :

$$
v A\langle\mathbf{u}\rangle+\langle B(\mathbf{u}, \mathbf{u})\rangle=\mathbf{f}_{P} .
$$

Proof. Let $\psi$ be a $C^{1}$ real-valued function with compact support on $\mathbb{R}$. For any $\mathbf{v} \in V$ and any wavenumber $\kappa$, the function $\mathbf{u} \mapsto \Phi(\mathbf{u})=\psi\left(\left(\mathbf{u}, \mathbf{v}_{\kappa_{1}, \kappa}\right)\right)$ is a cylindrical test function. Thus, $\Phi \in \mathcal{T}$ and

$$
\int_{H} \psi^{\prime}\left(\left(\mathbf{u}, \mathbf{v}_{\kappa_{1}, \kappa}\right)\right)\left\{\left(\mathbf{f}_{P}, \mathbf{v}_{\kappa_{1}, \kappa}\right)-v\left(A \mathbf{u}, \mathbf{v}_{\kappa_{1}, \kappa}\right)-b\left(\mathbf{u}, \mathbf{u}, \mathbf{v}_{\kappa_{1}, \kappa}\right)\right\} \mathrm{d} \mu(\mathbf{u})=0 .
$$

The terms inside the brackets are $\mu$-integrable, thanks to the condition (1) of finite mean enstrophy in the Definition 3.2 of stationary statistical solutions. 
Let $\psi^{\prime}$ converge pointwise to 1 while being uniformly bounded, so that at the limit we find

$$
\int_{H}\left\{\left(\mathbf{f}_{P}, \mathbf{v}_{\kappa_{1}, \kappa}\right)-v\left(A \mathbf{u}, \mathbf{v}_{\kappa_{1}, \kappa}\right)-b\left(\mathbf{u}, \mathbf{u}, \mathbf{v}_{\kappa_{1}, \kappa}\right)\right\} \mathrm{d} \mu(\mathbf{u})=0 .
$$

For each fixed $\mathbf{v} \in V$, we may let $\kappa$ go to infinity to find (since $\mu$ has finite enstrophy and a support bounded in $H$ ):

$$
\int_{H}\left\{\left(\mathbf{f}_{P}, \mathbf{v}\right)-v(A \mathbf{u}, \mathbf{v})-b(\mathbf{u}, \mathbf{u}, \mathbf{v})\right\} \mathrm{d} \mu(\mathbf{u})=0,
$$

which gives us the result.

We end this section with a result concerning the Grashof number $G^{*}$, which appears in the bound in $H$ for the weak attractor $\mathcal{A}_{w}$. Namely, we express $G^{*}$ in terms of the pressure gradient $P / L_{x}$ and other physical quantities:

Lemma 3.1. We have,

$$
G^{*}=\frac{\sqrt{3} L_{x}^{1 / 2} L_{y}^{1 / 2} h^{2}}{6 \pi^{1 / 2} \nu^{2}} \frac{P}{L_{x}}
$$

Proof. Since $\mathbf{f}_{P}=\left(P / L_{x}\right) \mathbf{e}_{\mathbf{1}}$, we have

$$
A^{-1} \mathbf{f}_{P}=\left(\frac{P}{2 L_{x}} z(h-z), 0,0\right) .
$$

Hence,

$$
\left|A^{-1 / 2} \mathbf{f}_{P}\right|_{0}^{2}=\left(\mathbf{f}_{P}, A^{-1} \mathbf{f}_{P}\right)=\int_{\Omega} \frac{P}{L_{x}} \frac{P}{2 L_{x}} z(h-z) \mathrm{d} x=\frac{L_{y} h^{3}}{12 L_{x}} P^{2} .
$$

Taking the square root of the equality above and substituting it in the definition (13) of the Grashof number gives us the result.

Remark 3.1. The vector-field $A^{-1} \mathbf{f}_{P}$ is directly related to the plane Poiseuille flow. In fact, the plane Poiseuille flow is precisely $\mathbf{u}=A^{-1} \mathbf{f}_{P} / v=\left(P z(h-z) / 2 v L_{x}, 0,0\right)$; see Section 7.

\section{Characteristic dimensions and nondimensional numbers}

The macroscopic characteristic length is considered to be $h$ and the macroscale characteristic wavenumber is $\kappa_{0}=1 / h$. The total mass of the fluid in the channel is $\rho_{0} L_{x} L_{y} h$, where $\rho_{0}$ denotes the uniform mass density of the fluid. Then, for a given stationary statistical solution $\mu$, the corresponding mean kinetic energy per unit mass and the mean energy dissipation rate per unit time and unit mass are given respectively by

$$
e=\frac{1}{2 L_{x} L_{y} h}\left\langle|\mathbf{u}|_{0}^{2}\right\rangle, \quad \epsilon=\frac{v}{L_{x} L_{y} h}\left\langle\|\mathbf{u}\|^{2}\right\rangle .
$$

The mean longitudinal bulk velocity is defined by

$$
U=\frac{1}{L_{y} h} \int_{H}\left(\int_{0}^{h} \int_{0}^{L_{y}} u_{1}(x, y, z) \mathrm{d} y \mathrm{~d} z\right) \mathrm{d} \mu(\mathbf{u}) ;
$$

note that this definition makes sense and the expression does not depend on $x$ due to the incompressibility and boundary conditions.

Thanks to the condition (3) of Definition 3.2 and to the divergence-free and boundary conditions, the mean longitudinal bulk velocity and the mean energy dissipation rate are related by

$$
\epsilon \leq \frac{P}{L_{x}} U
$$

Since we are assuming that $P, L_{x}>0$, it follows from the Reynolds equation (15) that $\mu$ cannot be a Dirac measure concentrated on the origin $\mathbf{u}=0$. Indeed, if that were the case, then both $\langle\mathbf{U}\rangle=0$ and $\langle B(\mathbf{u}, \mathbf{u})\rangle=0$, which would contradict the condition that $\mathbf{f}_{P}=\left(P / L_{x}\right) \mathbf{e}_{1} \neq 0$. Therefore, $\left\langle\|\mathbf{u}\|^{2}\right\rangle>0$, and it follows that

$$
\epsilon>0, \quad U>0 \text {. }
$$

With the velocity scale $U$ we may define the following Reynolds number

$$
R e=\frac{U h}{v}
$$


The dimensionless ratio of the applied pressure gradient to the square of the flow velocity scale is called the skin friction coefficient and it is given by

$$
C_{f}=\frac{h}{U^{2}} \frac{P}{L_{x}} .
$$

Now, suppose that $\mathbf{u}(\mathbf{x}, t)$ is a weak solution of the Navier-Stokes equations (1) with initial condition $\mathbf{u}_{0}(\mathbf{x})$. We define the finite-time average longitudinal bulk velocity by

$$
U_{T}=\frac{1}{L_{y} h} \frac{1}{T} \int_{0}^{T}\left(\int_{0}^{h} \int_{0}^{L_{y}} u_{1}(x, y, z, t) \mathrm{d} y \mathrm{~d} z\right) \mathrm{d} t .
$$

This expression is also well defined and independend of $x$ due to the incompressibility and boundary conditions and in this case we also have $U_{T}>0$.

In the sequel, we will also consider the similarly defined time-averaged dissipation rate

$$
\frac{v}{L_{x} L_{y} h}\left\langle\|\mathbf{u}\|^{2}\right\rangle_{T}=\frac{v}{L_{x} L_{y} h} \frac{1}{T} \int_{0}^{T}\|\mathbf{u}(t)\|^{2} \mathrm{~d} t,
$$

and the time-averaged kinetic energy

$$
\frac{1}{2 L_{x} L_{y} h}\left\langle|\mathbf{u}|_{0}^{2}\right\rangle_{T}=\frac{1}{2 L_{x} L_{y} h} \frac{1}{T} \int_{0}^{T}|\mathbf{u}(t)|_{0}^{2} \mathrm{~d} t .
$$

Of course the fact that these finite-time averages are bounded independently on $T$ does not guarantee that their long-time limits exist for $T \rightarrow \infty$.

\section{Time averages and stationary statistical solutions}

Since the usual limit of long-time averaged quantities may not exist, we aim to obtain eventual bounds for these time averaged quantities. In this section, we will establish, via generalized limits, a rigorous relationship between certain limits of these quantities and the stationary statistical solutions, see [12].

For example, suppose we are interested in estimating the upper limit of the time averaged longitudinal bulk velocity of a weak solution $\mathbf{u}(\mathbf{x}, t)$ of $(1)$ :

$$
\bar{U}=\frac{1}{L_{y} h} \limsup _{T \rightarrow \infty} \frac{1}{T} \int_{0}^{T}\left(\int_{0}^{L_{y}} \int_{0}^{h} u_{1}(x, y, z, t) \mathrm{d} y \mathrm{~d} z\right) \mathrm{d} t .
$$

Since an upper bound for the mean longitudinal bulk velocity $U_{\mu}$, associated with an arbitrary stationary statistical solution $\mu$, is derived in 6.1 , namely

$$
U_{\mu} \leq \frac{\sqrt{3} h^{2}}{6 v} \frac{P}{L_{x}}
$$

we may establish a direct relation between the time average longitudinal bulk velocity (24) and the mean longitudinal bulk velocity associated with a suitably defined stationary statistical solution $\mu_{0}$ (see Remark 5.1), such that

$$
\limsup _{T \rightarrow \infty} \frac{1}{T} \int_{0}^{T}\left(\int_{0}^{L_{y}} \int_{0}^{h} u_{1}(x, y, z, t) \mathrm{d} y \mathrm{~d} z\right) \mathrm{d} t=\int_{H}\left(\int_{0}^{L_{y}} \int_{0}^{h} u_{1}(x, y, z) \mathrm{d} y \mathrm{~d} z\right) \mathrm{d} \mu_{0}(\mathbf{u}),
$$

in such a way that we can give an upper bound to (24) using 6.1, obtaining

$$
\frac{1}{L_{y} h} \limsup _{T \rightarrow \infty} \frac{1}{T} \int_{0}^{T}\left(\int_{0}^{L_{y}} \int_{0}^{h} u_{1}(x, y, z, t) \mathrm{d} y \mathrm{~d} z\right) \mathrm{d} t=U_{\mu_{0}} \leq \frac{\sqrt{3} h^{2}}{6 v} \frac{P}{L_{x}} .
$$

This relation between long-time averages and stationary statistical solutions is realized via the notion of a generalized limit, which is defined as follows

Definition 5.1. A generalized limit is any linear functional, denoted $\operatorname{LiM}_{T \rightarrow \infty}$, defined on the space $\mathcal{B}([0, \infty))$ of all bounded real-valued functions on $[0, \infty)$ and satisfying

(1) $\operatorname{LIM}_{T \rightarrow \infty} g(T) \geq 0, \forall g \in \mathcal{B}([0, \infty))$ with $g(s) \geq 0, \forall s \geq 0$;

(2) $\operatorname{LIM}_{T \rightarrow \infty} g(T)=\lim _{T \rightarrow \infty} g(T), \forall g \in \mathcal{B}([0, \infty))$ such that the classical limit, denoted lim $T \rightarrow \infty$, exists. 
Remark 5.1. It is important for our argument below to recall that, given a particular $g_{0} \in \mathcal{B}([0, \infty))$ and a sequence $t_{j} \rightarrow \infty$ for which $g_{0}\left(t_{j}\right)$ converges to a number $l$, there exists a generalized limit $\operatorname{LIM}_{T \rightarrow \infty}$ satisfying $\operatorname{LiM}_{T \rightarrow \infty} g_{0}=l$; see [2,12]. In particular, for any $g_{0} \in \mathcal{B}([0, \infty))$ which does not converge to a limit as $t \rightarrow \infty$, we can find two generalized limits corresponding to the lower and upper limits of $g_{0}(t)$ as $t \rightarrow \infty$.

Proposition 5.1. Let $\varphi \in C\left(H_{w}\right)$. Suppose that for every stationary statistical solution $\mu$ the associated average of $\varphi$ satisfies $\langle\varphi\rangle \leq C_{1}$, for some constant $C_{1}$. Then, given a weak solution $\mathbf{w}(\mathbf{x}, t)$ defined on $[0, \infty)$, we have

$$
\limsup _{T \rightarrow \infty} \frac{1}{T} \int_{0}^{T} \varphi(\mathbf{w}(t)) \mathrm{d} t \leq C_{1} .
$$

Similarly, if for some constant $C_{2}$ we have $\langle\varphi\rangle \geq C_{2}$ for every stationary statistical solution, then

$$
\liminf _{T \rightarrow \infty} \frac{1}{T} \int_{0}^{T} \varphi(\mathbf{w}(t)) \mathrm{d} t \geq C_{2}
$$

Proof. We will prove inequality (27). Inequality (28) follows by a similar argument.

Let $\mathbf{w}_{0}=\mathbf{w}(0)$. Consider the set

$$
K_{w}=\left\{\mathbf{v} \in H ;|\mathbf{v}|^{2} \leq\left|\mathbf{w}_{0}\right|_{0}^{2}+\left|\mathbf{f}_{P}\right|_{0}^{2} / v^{2} \lambda_{1}^{2}\right\},
$$

endowed with the weak topology of $H . K_{w}$ is compact in $H_{w}$ and is such that $\mathbf{w}(t) \in K_{w}$, for all $t \geq 0$; see [12,22].

Let $\psi \in C\left(K_{w}\right)$. Since $K_{w}$ is compact, the function $t \mapsto \psi(\mathbf{w}(t))$ is continuous and bounded. Thus,

$$
g_{0}(t)=\frac{1}{t} \int_{0}^{t} \psi(\mathbf{w}(s)) \mathrm{d} s
$$

makes sense, and is continuous and bounded for $t \geq 0$. Therefore, its generalized limit is well defined, and by Remark 5.1, if we choose a subsequence $t_{j} \rightarrow \infty$ for which $g_{0}\left(t_{j}\right)$ converges to $\lim \sup _{t \rightarrow \infty} g_{0}(t)$, there exists a generalized limit $\operatorname{LiM}_{T \rightarrow \infty}$ satisfying

$$
\operatorname{LiM}_{T \rightarrow \infty} \frac{1}{T} \int_{0}^{T} \psi(\mathbf{w}(t)) \mathrm{d} t=\limsup _{t \rightarrow \infty} \frac{1}{t} \int_{0}^{t} \psi(\mathbf{w}(s)) \mathrm{d} s .
$$

Now, we relate this generalized time average with stationary statistical solutions. Since the weak solution $t \mapsto \mathbf{w}(t)$ belongs to the compact set $K_{w}$ in $H_{w}$, see [12], and since

$$
\psi \mapsto \operatorname{LiM}_{T \rightarrow \infty} \frac{1}{T} \int_{0}^{T} \psi(\mathbf{w}(t)) \mathrm{d} t
$$

is a positive linear functional on $\mathcal{C}\left(K_{w}\right)$, we use the Kakutani-Riesz Representation Theorem (see [25]) and conclude that there exists a measure $\mu_{0}$ on $H$ such that

$$
\operatorname{LiM}_{T \rightarrow \infty} \frac{1}{T} \int_{0}^{T} \psi(\mathbf{w}(\mathbf{x}, t)) \mathrm{d} t=\int_{H} \psi(\mathbf{u}) \mathrm{d} \mu_{0}(\mathbf{u}),
$$

for all $\psi \in C\left(K_{w}\right)$. It is shown in [12] that $\mu_{0}$ defined above is a stationary statistical solution.

Therefore, since $\left.\varphi\right|_{K_{w}} \in \mathcal{C}\left(K_{w}\right)$, and $\mu\left(H \backslash K_{w}\right)=0$, for every stationary statistical solution, (see [12]), and in particular for $\mu_{0}$, we conclude that

$$
\limsup _{t \rightarrow \infty} \frac{1}{t} \int_{0}^{t} \varphi(\mathbf{w}(x, t)) \mathrm{d} t=\int_{H} \varphi(\mathbf{u}) \mathrm{d} \mu_{0}(\mathbf{u}) .
$$

Thus, since $\langle\varphi\rangle \leq C_{1}$ for all stationary statistical solutions $\mu$, and in particular $\mu_{0}$, we have

$$
\limsup _{T \rightarrow \infty} \frac{1}{T} \int_{0}^{T} \varphi(\mathbf{w}(t)) \mathrm{d} t=\int_{H} \varphi(\mathbf{u}) \mathrm{d} \mu_{0}(\mathbf{u}) \leq C_{1}
$$

Now, returning to the mean longitudinal bulk velocity example, since

$$
\mathbf{u} \mapsto \int_{0}^{L_{y}} \int_{0}^{h} u_{1}(x, y, z) \mathrm{d} y \mathrm{~d} z
$$

belongs to $C\left(K_{w}\right)$, there exists a stationary statistical solution $\mu_{0}$ satisfying (25), which together with 6.1, yields the upper bound (26). 
Remark 5.2. Proposition 5.1 shows that every estimate involving the average of a continuous quantity on $C\left(K_{w}\right)$ can be stated as a superior or inferior limit of its time average.

This is true for the energy injection term, $\left(\mathbf{f}_{P}, \mathbf{u}\right)$, and also for the mean longitudinal bulk velocity $U$. However, we are also interested in estimating quantities involving $|\mathbf{u}|_{0}$ and $\|\mathbf{u}\|$, which are not weakly continuous. Fortunately, we are still able to estimate these quantities by approximating via Galerkin projections as shown in the next proposition.

Proposition 5.2. Let $\mathbf{w}(\mathbf{x}, t)$ be a weak solution of the NSE defined on $[0, \infty)$, and suppose that for every stationary statistical solution $\mu$, we have the following bounds

$$
C_{1} \leq \int_{H}|\mathbf{u}|_{0}^{2} \mathrm{~d} \mu_{0}(\mathbf{u}) \leq C_{2},
$$

and

$$
C_{3} \leq \int_{H}\|\mathbf{u}\|^{2} \mathrm{~d} \mu_{0}(\mathbf{u}) .
$$

Then, we also have

$$
\begin{aligned}
& \liminf _{T \rightarrow \infty} \frac{1}{T} \int_{0}^{T}|\mathbf{w}(t)|_{0}^{2} \mathrm{~d} t \geq C_{1}, \\
& \limsup _{T \rightarrow \infty} \frac{1}{T} \int_{0}^{T}|\mathbf{w}(t)|_{0}^{2} \mathrm{~d} t \leq C_{2},
\end{aligned}
$$

and

$$
\liminf _{T \rightarrow \infty} \frac{1}{T} \int_{0}^{T}\|\mathbf{w}(t)\|^{2} \mathrm{~d} t \geq C_{3} .
$$

Proof. Since $\left|P_{\kappa} \mathbf{u}\right|_{0},\left\|P_{\kappa} \mathbf{u}\right\|$ belong to $C\left(K_{w}\right)$, as functions of $\mathbf{u}$, where $P_{\kappa}$ are the usual Galerkin projectors associated with the wavenumbers lower than or equal to $\kappa$ we have by (29), that, given a stationary statistical solution $\mu_{0}$ generated by a generalized time average, the following equations are valid

$$
\operatorname{LIM}_{T \rightarrow \infty} \frac{1}{T} \int_{0}^{T}\left|P_{\kappa} \mathbf{w}(t)\right|_{0}^{2} \mathrm{~d} t=\int_{H}\left|P_{\kappa} \mathbf{u}\right|_{0}^{2} \mathrm{~d} \mu_{0}(\mathbf{u}),
$$

and

$$
\operatorname{LiM}_{T \rightarrow \infty} \frac{1}{T} \int_{0}^{T}\left\|P_{\kappa} \mathbf{w}(t)\right\|^{2} \mathrm{~d} t=\int_{H}\left\|P_{\kappa} \mathbf{u}\right\|^{2} \mathrm{~d} \mu_{0}(\mathbf{u}) .
$$

Now, since

$$
|\mathbf{w}(t)|_{0}^{2}-\left|P_{\kappa} \mathbf{w}(t)\right|_{0}^{2}=\left|Q_{\kappa} \mathbf{w}(t)\right|_{0}^{2} \leq \kappa^{-2}\|\mathbf{w}(t)\|^{2},
$$

and

$$
\frac{1}{T} \int_{0}^{T}\|\mathbf{w}(t)\|^{2} \mathrm{~d} t \leq C<\infty,
$$

where $C$ is independent of $T[5,21]$, we have by the usual properties of the generalized limits that

$$
\begin{aligned}
& \operatorname{Lim}_{T \rightarrow \infty} \frac{1}{T} \int_{0}^{T}|\mathbf{w}(t)|^{2} \mathrm{~d} t-\operatorname{LIM}_{T \rightarrow \infty} \frac{1}{T} \int_{0}^{T}\left|P_{\kappa} \mathbf{w}(t)\right|^{2} \mathrm{~d} t \\
& \quad \leq \kappa^{-2} \operatorname{Lim}_{T \rightarrow \infty} \frac{1}{T} \int_{0}^{T}\|\mathbf{w}(t)\|^{2} \mathrm{~d} t \\
& \quad \leq \kappa^{-2} \limsup \frac{1}{T} \int_{0}^{T}\|\mathbf{w}(t)\|^{2} \mathrm{~d} t \leq C \kappa^{-2} \rightarrow 0, \quad \kappa \rightarrow \infty .
\end{aligned}
$$

Thus, as indicated in Remark 5.1, considering the generalized limit $\operatorname{LIM}_{T \rightarrow \infty}$ that extends the left hand side of (32), we have

$$
\begin{aligned}
\liminf _{T \rightarrow \infty} \frac{1}{T} \int_{0}^{T}|\mathbf{w}(t)|_{0}^{2} \mathrm{~d} t & =\operatorname{Lim}_{T \rightarrow \infty} \frac{1}{T} \int_{0}^{T}|\mathbf{w}(t)|_{0}^{2} \mathrm{~d} t \\
& \geq \operatorname{Lim}_{T \rightarrow \infty} \frac{1}{T} \int_{0}^{T}\left|P_{\kappa} \mathbf{w}(t)\right|^{2} \mathrm{~d} t=\int_{H}\left|P_{\kappa} \mathbf{u}\right|^{2} \mathrm{~d} \mu_{0}(\mathbf{u}),
\end{aligned}
$$


for any wavenumber $\kappa$, assuming $\left|P_{\kappa} \mathbf{w}(t)\right| \leq|\mathbf{w}(t)|$ for all $\kappa$. Using the Monotone Convergence Theorem and hypothesis (30), we obtain

$$
\lim _{\kappa \rightarrow \infty} \int_{H}\left|P_{\kappa} \mathbf{u}\right|^{2} \mathrm{~d} \mu_{0}(\mathbf{u})=\int_{H}|\mathbf{u}|^{2} \mathrm{~d} \mu_{0}(\mathbf{u}) \geq C_{1} .
$$

Thus,

$$
\liminf _{T \rightarrow \infty} \frac{1}{T} \int_{0}^{T}|\mathbf{w}(t)|_{0}^{2} \mathrm{~d} t \geq C_{1}
$$

Bound (33) follows in a similar way.

Now, we will prove (34). Consider the generalized limit, $\operatorname{LIM}_{T \rightarrow \infty}$, that extends the left hand side of (34), and notice that

$$
\|\mathbf{w}(t)\| \geq\left\|P_{\kappa} \mathbf{w}(t)\right\| \text {. }
$$

Then, we have

$$
\begin{aligned}
& \liminf _{T \rightarrow \infty} \frac{1}{T} \int_{0}^{T}\|\mathbf{w}(t)\|_{0}^{2} \mathrm{~d} t=\operatorname{LiM}_{T \rightarrow \infty} \frac{1}{T} \int_{0}^{T}\|\mathbf{w}(t)\|_{0}^{2} \mathrm{~d} t \\
& \geq \lim _{\kappa \rightarrow \infty} \operatorname{LiM}_{T \rightarrow \infty} \frac{1}{T} \int_{0}^{T}\left\|P_{\kappa} \mathbf{w}(t)\right\|^{2} \mathrm{~d} t \\
& \quad=\lim _{\kappa \rightarrow \infty} \int_{H}\left\|P_{\kappa} \mathbf{u}\right\|^{2} \mathrm{~d} \mu_{0}(\mathbf{u})=\int_{H}\|\mathbf{u}\|^{2} \mathrm{~d} \mu_{0}(\mathbf{u}) \geq C_{3},
\end{aligned}
$$

where, again, the last equality in the expression above follows from the Monotone Convergence Theorem.

Remark 5.3. Propositions 5.1 and 5.2 show that, except for Proposition 7.2 and Theorem 7.3 below, every estimate in the sequel about physical mean quantities, defined in terms of ensemble averages associated with stationary statistical solutions, can be stated as estimates for the superior or inferior limit of time averages of such quantities. The reason why these results do not apply to Proposition 7.2 and Theorem 7.3 is that they involve an upper bound for $\|\mathbf{u}\|$, which is not considered by the propositions above. However, they can still be stated in terms of their corresponding time averages as seen in Remark 7.1.

\section{Estimates on the mean longitudinal bulk velocity and on the skin friction coefficient}

We start by deriving an upper bound for the mean longitudinal bulk velocity $U$, which implies a lower bound on the skin friction coefficient $C_{f}$.

Theorem 6.1. For every stationary statistical solution, the mean longitudinal bulk velocity $U$ satisfies

$$
U \leq \frac{h^{2}}{12 \pi v} \frac{P}{L_{x}}
$$

and the skin friction coefficient satisfies

$$
C_{f} \geq \frac{12}{R e} \text {. }
$$

Proof. It follows directly from the definition of $U$ that

$$
U=\frac{1}{L_{x} L_{y} h}\left\langle\left(\mathbf{u}, \mathbf{e}_{1}\right)\right\rangle \leq \frac{1}{L_{x} L_{y} h}\left|A^{-1 / 2} \mathbf{e}_{1}\right|_{0}\langle\|\mathbf{u}\|\rangle \leq \frac{1}{L_{x} L_{y} h}\left|A^{-1 / 2} \mathbf{e}_{1}\right|_{0}\left\langle\|\mathbf{u}\|^{2}\right\rangle^{1 / 2} .
$$

Similarly to the computations in (17) one can check that

$$
\left|A^{-1 / 2} \mathbf{e}_{1}\right|_{0}^{2}=\frac{L_{x} L_{y} h^{3}}{12},
$$

so that

$$
U \leq \frac{1}{\sqrt{12}} \frac{h^{1 / 2}}{L_{x}^{1 / 2} L_{y}^{1 / 2}}\left\langle\|\mathbf{u}\|^{2}\right\rangle^{1 / 2} \leq \frac{h}{\sqrt{12} v^{1 / 2}} \epsilon^{1 / 2} .
$$

Using (19) and (42) we obtain the desired upper bound for $U$. 
Using this estimate and the definition of the skin friction coefficient we find that

$$
C_{f}=\frac{P h}{L_{x} U^{2}} \geq \frac{P h}{L_{x} U} \frac{12 \pi v L_{x}}{h^{2} P}=\frac{12 v}{h U},
$$

and the result follows from the definition (21) of the Reynolds number.

Now, we give a lower bound estimate for the mean longitudinal bulk velocity $U$, following the calculations of [4], but avoiding using an equation for the fluctuation $\mathbf{v}$.

Proposition 6.1. For every stationary statistical solution, the mean longitudinal bulk velocity $U$ satisfies

$$
U \geq \sup \left\{\frac{h^{2}}{12 v} \frac{P}{L_{x}}-\frac{v}{h} \frac{L_{x}}{P} \int_{0}^{h}\left(U_{1}^{\prime}(z)-\frac{1}{2 v} \frac{P}{L_{x}}(h-2 z)\right)^{2} \mathrm{~d} z ; \mathbf{U} \in \mathcal{U}\right\},
$$

where

$$
\mathcal{U}=\left\{\mathbf{U} \in V ; \mathbf{U}=\left(U_{1}(z), 0,0\right), U_{1} \in H_{0}^{1}(0, h),\left\langle H_{\mathbf{U}}(\mathbf{v})\right\rangle \geq 0, \forall \mathbf{v} \in V\right\},
$$

and

$$
H_{\mathbf{U}}(\mathbf{v})=\frac{v}{2}\|\mathbf{v}\|^{2}+b(\mathbf{v}, \mathbf{U}, \mathbf{v})
$$

Proof. Let $\mathbf{U} \in V$ be of the form $\mathbf{U}(x, y, z)=\left(U_{1}(z), 0,0\right)$. We have

$$
\left\langle\|\mathbf{u}-\mathbf{U}\|^{2}\right\rangle=\left\langle\|\mathbf{u}\|^{2}\right\rangle-2\langle((\mathbf{u}, \mathbf{U}))\rangle+\|\mathbf{U}\|^{2} .
$$

Since $\mathbf{U} \in V$ is fixed we can multiply it with the Reynolds equations (15) and obtain

$$
v\langle((\mathbf{u}, \mathbf{U}))\rangle=\left(\mathbf{f}_{P}, \mathbf{U}\right)-\langle b(\mathbf{u}, \mathbf{u}, \mathbf{U})\rangle .
$$

Substituting (45) into (44), we obtain

$$
v\left\langle\|\mathbf{u}\|^{2}\right\rangle=v\left\langle\|\mathbf{u}-\mathbf{U}\|^{2}\right\rangle+2\left(\left(\mathbf{f}_{P}, \mathbf{U}\right)-\langle b(\mathbf{u}, \mathbf{u}, \mathbf{U})\rangle\right)-v\left\langle\|\mathbf{U}\|^{2}\right\rangle .
$$

Since $\mathbf{U}(x, y, z)=\left(U_{1}(z), 0,0\right)$, by the anti-symmetry property of the trilinear term, $b(\mathbf{u}, \mathbf{u}, \mathbf{U})=-b(\mathbf{u}, \mathbf{U}, \mathbf{u})$, and by (19) we have

$$
\begin{aligned}
L_{x} L_{y} h U & \geq \frac{\nu L_{x}}{P}\left\langle\|\mathbf{u}\|^{2}\right\rangle \\
& =\frac{2 L_{x}}{P}\left\langle\nu \frac{\|\mathbf{u}-\mathbf{U}\|^{2}}{2}+b(\mathbf{u}, \mathbf{U}, \mathbf{u})\right\rangle+2 \frac{L_{x}}{P}\left(\mathbf{f}_{P}, \mathbf{U}\right)-\frac{\nu L_{x}}{P}\|\mathbf{U}\|^{2} .
\end{aligned}
$$

Due to the form of $\mathbf{U}$, we have $b(\mathbf{U}, \mathbf{U}, \mathbf{u})=0$. The orthogonality property implies

$$
b(\mathbf{u}-\mathbf{U}, \mathbf{U}, \mathbf{U})=0 .
$$

Thus,

$$
b(\mathbf{u}, \mathbf{U}, \mathbf{u})=b(\mathbf{u}-\mathbf{U}, \mathbf{U}, \mathbf{u}-\mathbf{U}), \quad \forall \mathbf{u} \in V .
$$

Since $\mu$ is carried by $V$, we find

$$
\left(L_{x} L_{y} h\right) U=\frac{2 L_{x}}{P}\left\langle\nu \frac{\|\mathbf{u}-\mathbf{U}\|^{2}}{2}+b(\mathbf{u}-\mathbf{U}, \mathbf{U}, \mathbf{u}-\mathbf{U})\right\rangle+\frac{2 L_{x}}{P}\left(\mathbf{f}_{P}, \mathbf{U}\right)-\frac{v L_{x}}{P}\|\mathbf{U}\|^{2} .
$$

Thus, considering only background flows $\mathbf{U}$ such that $\left\langle H_{\mathbf{U}}(\mathbf{v})\right\rangle \geq 0$ for all $\mathbf{v} \in V$, we have

$$
\left(L_{x} L_{y} h\right) U \geq \frac{2 L_{x}}{P}\left(\mathbf{f}_{P}, \mathbf{U}\right)-\frac{v L_{x}}{P}\|\mathbf{U}\|^{2} .
$$

Substituting for $\mathbf{U}=\left(U_{1}(z), 0,0\right)$, we obtain

$$
U \geq \frac{2}{h} \int_{0}^{h} U_{1}(z) \mathrm{d} z-\frac{v}{h} \frac{L_{x}}{P} \int_{0}^{h} U_{1}^{\prime}(z)^{2} \mathrm{~d} z .
$$

Expanding the integrand in (43) and using integration by parts one can see that the expression in (43) is equal to the right hand side above, and this completes the proof. 
Remark 6.1. The result in Proposition 6.1 was given in [4] in the context of long time averages. It was obtained from a derivation of an energy equation for the fluctuation variable $\mathbf{v}=\mathbf{u}-\mathbf{U}$ :

$$
\frac{1}{2} \frac{\mathrm{d}}{\mathrm{d} t}|\mathbf{v}|_{0}^{2}+v\|\mathbf{v}\|^{2}+v((\mathbf{v}, \mathbf{U}))+b(\mathbf{U}, \mathbf{U}, \mathbf{v})+b(\mathbf{v}, \mathbf{U}, \mathbf{v})=\left(\mathbf{f}_{P}, \mathbf{v}\right)
$$

and by considering an energy equation for $\mathbf{u}$ :

$$
\frac{1}{2} \frac{\mathrm{d}}{\mathrm{d} t}|\mathbf{u}|_{0}^{2}+v\|\mathbf{u}\|^{2}=\left(L_{y} h\right) P U .
$$

Taking the long time average in both sides of (52), considering the same hypothesis for $\mathbf{U}$, and substituting it into (51), the corresponding result for long time averages was obtained. However, since we want to consider any stationary statistical solutions and general weak solutions of the Navier-Stokes equations, we must treat the fluctuation component carefully and avoid the energy Eq. (51) and the equality in (52). The slightly modified and in fact simpler proof presented in Proposition 6.1 achieves this aim.

Applying the variational estimate in Proposition 6.1 with a suitable background flow $\mathbf{U}$ in $\mathcal{U}$ we obtain the following explicit bounds for the longitudinal bulk velocity and the skin-friction coefficient.

Theorem 6.2. For every stationary statistical solution, the mean longitudinal bulk velocity $U$ and the skin friction coefficient $C_{f}$ satisfy

$$
U \geq \begin{cases}\frac{2}{27} \frac{h^{2}}{v} \frac{P}{L_{x}}, & \text { if } 0<\frac{P}{L_{x}} \leq \frac{27 \sqrt{2} \pi^{2} v^{2}}{4 h^{3}}, \\ \frac{2^{5 / 4} \pi}{3^{3 / 2}} h^{1 / 2}\left(\frac{P}{L_{x}}\right)^{1 / 2}-\frac{\sqrt{2} \pi^{2}}{2} \frac{v}{h}, & \text { if } \frac{P}{L_{x}}>\frac{27 \sqrt{2} \pi^{2} v^{2}}{4 h^{3}},\end{cases}
$$

and

$$
C_{f} \leq \begin{cases}\frac{27}{2} \frac{1}{R e}, & \text { if } 0<\frac{P}{L_{x}} \leq \frac{27 \sqrt{2} \pi^{2} v^{2}}{4 h^{3}} \\ \frac{27 \sqrt{2}}{8 \pi^{2}}\left(1+\frac{\sqrt{2} \pi^{2}}{2} \frac{1}{R e}\right)^{2}, & \text { if } \frac{P}{L_{x}} \geq \frac{27 \sqrt{2} \pi^{2} v^{2}}{4 h^{3}}\end{cases}
$$

Proof. Following Constantin and Doering [4], consider the background-flow of the form $\mathbf{U}(x, y, z)=\left(U_{1}(z), 0,0\right)$ with

$$
U_{1}(z)= \begin{cases}\frac{V}{\delta} z, & 0 \leq z \leq \delta \\ V, & \delta \leq z \leq h-\delta \\ \frac{V}{\delta}(h-z), & h-\delta \leq z \leq h .\end{cases}
$$

We will verify that for appropriate choices of $V$ and $\delta$ this flow satisfies the spectral constraint $H_{\mathbf{U}}(\mathbf{v}) \geq 0$, for all $\mathbf{v} \in V$. Noticing that

$$
H_{\mathbf{U}}(\mathbf{v})=\frac{v}{2}\|\mathbf{v}\|^{2}+\int_{0}^{h} \int_{0}^{L_{y}} \int_{0}^{L_{x}} U_{1}^{\prime}(z) v_{1}(\mathbf{x}) v_{3}(\mathbf{x}) \mathrm{d} x \mathrm{~d} y \mathrm{~d} z
$$

we bound the integral of $U_{1}^{\prime}(z) v_{1} v_{3}$ in terms of $\delta$ and $\|\mathbf{v}\|^{2}$. First, we divide this integral into two parts, one from 0 to $\delta$, and the other from $h-\delta$ to $h$.

In order to bound the first integral, consider the spaces $\tilde{H}=L^{2}(0, \delta)$, with the usual $L^{2}$ inner product, and $\tilde{V}=$ $\left\{\mathbf{u} \in H^{1}(0, \delta) ; u(0)=0\right\}$, with the inner product $((u, v))=\int_{0}^{\delta} u^{\prime}(z) v^{\prime}(z) \mathrm{d} z$. Consider the operator $\tilde{A}: \tilde{V} \rightarrow \tilde{H}$ defined by

$$
(\tilde{A} u, v)=((u, v)), \quad \forall v \in \tilde{V}
$$

and $D(\tilde{A})=\{u \in \tilde{V} ; \tilde{A} u \in \tilde{H}\}$. One can show that $\tilde{A}$ is self-adjoint and invertible, with a compact inverse, and that the smallest associated eigenvalue is $\tilde{\lambda}_{1}=\pi^{2} / 4 \delta^{2}$. Therefore,

$$
\tilde{\lambda}_{1} \int_{0}^{\delta}|u(z)|^{2} \mathrm{~d} z \leq \int_{0}^{\delta}\left|\frac{\partial u(z)}{\partial z}\right|^{2} \mathrm{~d} z .
$$


A similar statement can be made for the integral between $h-\delta$ and $h$. Thus, the integral of $U^{\prime}(z) v_{1} v_{3}$ can be estimated in the following way

$$
\begin{aligned}
\left|\int_{0}^{L_{x}} \int_{0}^{L_{y}} \int_{0}^{h} U^{\prime}(z) v_{1} v_{3} \mathrm{~d} x \mathrm{~d} y \mathrm{~d} z\right| & \leq \frac{V}{\delta}\left|\int_{0}^{L_{x}} \int_{0}^{L_{y}} \int_{0}^{\delta} v_{1} v_{3} \mathrm{~d} x \mathrm{~d} y \mathrm{~d} z-\int_{0}^{L_{x}} \int_{0}^{L_{y}} \int_{h-\delta}^{h} v_{1} v_{3} \mathrm{~d} x \mathrm{~d} y \mathrm{~d} z\right| \\
& \leq \frac{V}{\delta} \int_{0}^{L_{x}} \int_{0}^{L_{y}} \int_{0}^{\delta} \alpha \frac{\left|v_{1}\right|^{2}}{2}+\frac{\left|v_{3}\right|^{2}}{2 \alpha} \mathrm{d} x \mathrm{~d} y \mathrm{~d} z+\frac{V}{\delta} \int_{0}^{L_{x}} \int_{0}^{L_{y}} \int_{h-\delta}^{h} \alpha \frac{\left|v_{1}\right|^{2}}{2}+\frac{\left|v_{3}\right|^{2}}{2 \alpha} \mathrm{d} x \mathrm{~d} y \mathrm{~d} z \\
& \leq \frac{2 V \delta}{\pi^{2}}\left(\alpha\left|\frac{\partial v_{1}}{\partial z}\right|_{0}^{2}+\frac{1}{\alpha}\left|\frac{\partial v_{3}}{\partial z}\right|_{0}^{2}\right) \\
& \leq \frac{2 V \delta}{\pi^{2}}\left(\alpha\left|\frac{\partial v_{1}}{\partial z}\right|_{0}^{2}+\frac{1}{2 \alpha}\left(\left|\frac{\partial v_{3}}{\partial z}\right|_{0}^{2}+\left|\frac{\partial v_{1}}{\partial x}\right|_{0}^{2}+\left|\frac{\partial v_{2}}{\partial y}\right|_{0}^{2}+\left|\frac{\partial v_{1}}{\partial y}\right|_{0}^{2}+\left|\frac{\partial v_{2}}{\partial x}\right|_{0}^{2}\right)\right)
\end{aligned}
$$

The last step above follows from the inequality below, given in [3]:

$$
\left|\frac{\partial v_{3}}{\partial z}\right|_{0}^{2} \leq \frac{1}{2}\left(\left|\frac{\partial v_{3}}{\partial z}\right|_{0}^{2}+\left|\frac{\partial v_{1}}{\partial x}\right|_{0}^{2}+\left|\frac{\partial v_{2}}{\partial y}\right|_{0}^{2}+\left|\frac{\partial v_{1}}{\partial y}\right|_{0}^{2}+\left|\frac{\partial v_{2}}{\partial x}\right|_{0}^{2}\right),
$$

which is valid for divergence-free vector fields $\mathbf{v}=\left(v_{1}, v_{2}, v_{3}\right)$ in $V$.

Thus, choosing $\alpha=\sqrt{2} / 2$, we have

$$
\left|\int_{0}^{L_{x}} \int_{0}^{L_{y}} \int_{0}^{h} U_{1}^{\prime}(z) v_{1} v_{3} \mathrm{~d} x \mathrm{~d} y \mathrm{~d} z\right| \leq \frac{\sqrt{2}}{\pi^{2}} V \delta\|\mathbf{v}\|^{2} .
$$

Hence, $H_{\mathbf{U}}(\mathbf{v})$ is bounded from below by

$$
H_{\mathbf{U}}(\mathbf{v}) \geq\left(\frac{v}{2}-\frac{\sqrt{2}}{\pi^{2}} V \delta\right)\|\mathbf{v}\|^{2}
$$

Therefore, $H_{\mathbf{U}}$ is non-negative if $\delta \leq v \pi^{2} / 2 \sqrt{2} V$, with $V$ sufficiently large to fulfill the compatibility hypothesis $\delta \leq h / 2$. Hence, we can use this $\mathbf{U}$ in (50) and we obtain a lower bound for $U$ :

$$
U \geq \frac{2}{h}\left(h V-\delta V-\frac{\nu L_{x} V^{2}}{\delta P}\right) .
$$

We maximize the lower bound above, respecting the compatibility hypotheses, with the following choices of $V$ and $\delta$ :

$$
V=\frac{\pi h^{1 / 2}}{3^{1 / 2} 2^{3 / 4}} \frac{P^{1 / 2}}{L_{x}^{1 / 2}}, \quad \delta=\frac{3^{1 / 2} v}{2^{3 / 4} h^{1 / 2}} \frac{L_{x}^{1 / 2}}{P^{1 / 2}} \quad \text { if } \frac{P}{L_{x}}>\frac{27 \sqrt{2} \pi^{2} v^{2}}{4 h^{3}}
$$

and

$$
V=\frac{h^{2}}{9 v} \frac{P}{L_{x}}, \quad \delta=\frac{h}{3} \quad \text { if } \frac{P}{L_{x}} \leq \frac{27 \sqrt{2} \pi^{2} v^{2}}{4 h^{3}} .
$$

The result (53) follows immediately from the substitution of (57) and (58) into (56). Then, (53) can be applied to yield (54) in much the same was as done in the proof of Theorem 6.1 .

Remark 6.2. Theorem 6.2 gives a uniform upper bound estimate for the skin friction coefficient for high Reynolds numbers. As pointed out in [4], even though this constant upper bound estimate is predicted by the Kolmogorov theory of homogeneous turbulence, it is known from experiments that corrections are necessary for turbulence in the presence of walls, see also [3,18, 24]. Actually, closure approximation theories establish the following logarithmic friction law which has been confirmed by highReynolds-number pipe flow experiments:

$$
C_{f} \sim \frac{1}{(\ln R e)^{2}} .
$$

Thus, we conclude that while empirical arguments and experimental data predict a logarithmic friction law, our rigorous mathematical bounds can only assert that

$$
\frac{12}{R e} \leq C_{f} \leq \frac{27 \sqrt{2}}{8 \pi^{2}}+O\left(\frac{1}{R e^{2}}\right) .
$$

The lower bound for $C_{f}$ is precisely the skin friction coefficient for the plane Poiseuille flow; see Section 8 . 
Remark 6.3. Note also that for high-Reynolds-number flows, the characteristic background velocity which leads to the estimate above is of the order of

$$
V \sim h^{1 / 2}\left(\frac{P}{L_{x}}\right)^{1 / 2}
$$

while the corresponding "boundary layer" length is of the order of

$$
\delta \sim \frac{v}{h^{1 / 2}}\left(\frac{L_{x}}{P}\right)^{1 / 2}
$$

which seems to be consistent with the characteristics of the mean turbulent flow as observed in experiments and obtained from heuristic arguments (see e.g. [24]).

\section{Other estimates}

We start by deriving a lower bound for the energy dissipation rate $\epsilon$.

Theorem 7.1. For every stationary statistical solution, the energy dissipation rate satisfies

$$
\epsilon \geq \begin{cases}\frac{2}{27} \frac{h^{2}}{v}\left(\frac{P}{L_{x}}\right)^{2}, & \text { if } 0<\frac{P}{L_{x}} \leq \frac{27 \sqrt{2} \pi^{2} v^{2}}{4 h^{3}}, \\ \frac{2^{5 / 4} \pi}{3^{3 / 2}} h^{1 / 2}\left(\frac{P}{L_{x}}\right)^{3 / 2}-\frac{\sqrt{2} \pi^{2}}{2} \frac{v}{h} \frac{P}{L_{x}}, & \text { if } \frac{P}{L_{x}}>\frac{27 \sqrt{2} \pi^{2} v^{2}}{4 h^{3}} .\end{cases}
$$

Proof. The result follows from noticing that the estimate (47) obtained in Proposition 6.1 is actually a lower bound for $\left\langle\|\mathbf{u}\|^{2}\right\rangle$, and, therefore, we can follow the subsequent calculations in the exact same way with this term instead of $U$.

Now, we give a lower bound on the mean kinetic energy.

Theorem 7.2. For every stationary statistical solution, the mean kinetic energy e satisfies

$$
e \geq \frac{h}{6} \frac{P}{L_{x}}-\frac{4 v^{2}}{h^{2}}\left(1+\frac{h^{3}}{12 v^{2}} \frac{P}{L_{x}}\right)^{1 / 2}+\frac{4 v^{2}}{h^{2}} .
$$

Proof. Taking the inner product with $A^{-1} \mathbf{f}_{P}$ in the Reynolds equation yields

$$
\left|A^{-1 / 2} \mathbf{f}_{P}\right|_{0}^{2}=v\left\langle\left(\mathbf{u}, \mathbf{f}_{P}\right)\right\rangle+b\left(\mathbf{u}, \mathbf{u}, A^{-1} \mathbf{f}_{P}\right) \leq v\left\langle|\mathbf{u}|_{0}\right\rangle\left|\mathbf{f}_{P}\right|_{0}+\left\langle\left|b\left(\mathbf{u}, A^{-1} \mathbf{f}_{P}, \mathbf{u}\right)\right|\right\rangle,
$$

and since

$$
\begin{aligned}
\left\langle\mid b\left(\mathbf{u}, A^{-1} \mathbf{f}_{P}, \mathbf{u}\right) \|\right\rangle & =\left\langle\left|\int_{\Omega} u_{3}\left(\frac{\partial}{\partial z} \frac{P}{2 L_{x}} z(h-z)\right) u_{1} \mathrm{~d} x\right|\right\rangle \\
& \leq \frac{P}{2 L_{x}}\left\langle\int_{\Omega}|h-2 z|\left|u_{3}\right|\left|u_{1}\right| \mathrm{d} x\right\rangle \\
& \leq \frac{P h}{4 L_{x}}\left\langle\int_{\Omega}\left|u_{3}\right|^{2}+\left|u_{1}\right|^{2} \mathrm{~d} x\right\rangle \\
& \leq \frac{P h}{4 L_{x}}\left\langle|\mathbf{u}|_{0}^{2}\right\rangle,
\end{aligned}
$$

we find from (63) that

$$
\begin{aligned}
\left|A^{-1 / 2} \mathbf{f}_{P}\right|_{0}^{2} & \leq v\left|\mathbf{f}_{P}\right|\left\langle|\mathbf{u}|_{0}^{2}\right\rangle^{1 / 2}+\left\langle\left|b\left(\mathbf{u}, A^{-1} \mathbf{f}_{P}, \mathbf{u}\right)\right|_{0}\right\rangle \\
& \leq v \frac{P L_{y}^{1 / 2} h^{1 / 2}}{L_{x}^{1 / 2}}\left\langle|\mathbf{u}|_{0}^{2}\right\rangle^{1 / 2}+P \frac{h}{4 L_{x}}\left\langle|\mathbf{u}|_{0}^{2}\right\rangle .
\end{aligned}
$$

Then, using (17), we have

$$
\frac{L_{y} h^{3}}{12 L_{x}} P \leq \frac{v}{L_{x}}\left\langle|\mathbf{u}|_{0}^{2}\right\rangle^{1 / 2}+\frac{h}{4 L_{x}}\left\langle|\mathbf{u}|_{0}^{2}\right\rangle,
$$


which is of the form $a r^{2}+b r+c \geq 0$ for $r=\left\langle\|\mathbf{u}\|^{2}\right\rangle^{1 / 2}, a=h / 4 L_{x}, \quad b=v / L_{x}, \quad c=\left(L_{y} h^{3}\right) / 12 L_{x}$. It gives us $r^{2} \geq b^{2} / 2 a^{2}-b\left(b^{2}+4 a c\right)^{1 / 2} / a+c / a$, which implies

$$
\left\langle|\mathbf{u}|_{0}^{2}\right\rangle \geq \frac{h^{2} L_{y}}{3} P-\frac{8 v L_{x}}{h^{2}}\left(\frac{v^{2} L_{y}^{2} h^{2}}{P}+\frac{L_{y}^{2} h^{5}}{12 L_{x}}\right)^{1 / 2} P^{1 / 2}+\frac{8 v^{2} L_{x} L_{y}}{h} .
$$

Divide this expression by $2 L_{x} L_{y} h$ to obtain the result.

Theorem 7.3. For every stationary statistical solution, the energy dissipation rate $\epsilon$ satisfies

$$
\epsilon \leq \frac{h^{2}}{12 v}\left(\frac{P}{L_{x}}\right)^{2}
$$

Proof. This follows directly from inequalities (19) and 6.1.

Proposition 7.1. For every stationary statistical solution, the mean kinetic energy e satisfies

$$
e \leq \frac{h^{4}}{24 \pi^{2} v^{2}}\left(\frac{P}{L_{x}}\right)^{2}
$$

Proof. This follows directly from (67) and the Poincaré inequality (6).

Now, we state a partial rigorous confirmation of the Kolmogorov dissipation law in terms of $U$.

Proposition 7.2. For every stationary statistical solution, and sufficiently large pressure gradient $P / L_{x}$, namely $P / L_{x} \geq$ $27 \sqrt{2} \pi^{2} v^{2} / 4 h^{3}$, the associated energy dissipation rate satisfies

$$
\epsilon \leq\left(\frac{3}{2^{5 / 2} \pi^{2}}+\frac{27 \pi}{4} \frac{1}{R e}+\frac{27 \pi^{2}}{2^{7 / 2}} \frac{1}{R e^{2}}\right) \frac{U^{3}}{h} .
$$

Proof. Taking the square of both sides of the second inequality in (53), we have

$$
P \leq \frac{3 L_{x}}{2^{5 / 2} \pi^{2} h} U^{2}+\frac{27 L_{x} \pi v}{4 h^{2}} U+\frac{27 \pi^{2} L_{x} v^{2}}{2^{7 / 2} h^{3}} .
$$

Substituting (70) into (19), we obtain the result.

Remark 7.1. Note that we cannot invoke Proposition 5.1 nor Proposition 5.2 to state the results (69) and (67) in terms of their time averages. However, we can improve these results as follows.

Let $\mathbf{u}(\mathbf{x}, t)$ be a weak solution of the Navier-Stokes equations. It follows from the classical energy inequality for weak solutions of the NSE defined on $[0, \infty)$, see $[5,21]$, that

$$
\limsup _{T \rightarrow \infty} \frac{1}{T}\left(v \int_{0}^{T}\|\mathbf{u}(s)\|^{2} \mathrm{~d} s-\int_{0}^{T}\left(\mathbf{f}_{P}, \mathbf{u}(s)\right) \mathrm{d} s\right) \leq \limsup _{T \rightarrow \infty} \frac{1}{T}\left(\frac{1}{2}|\mathbf{u}(0)|_{0}^{2}-\frac{1}{2}|\mathbf{u}(T)|_{0}^{2}\right)=0 .
$$

Thus,

$$
\limsup _{T \rightarrow \infty} \frac{v}{T} \int_{0}^{T}\|\mathbf{u}(s)\|^{2} \mathrm{~d} s \leq \liminf _{T \rightarrow \infty} \frac{1}{T} \int_{0}^{T}\left(\mathbf{f}_{P}, \mathbf{u}(s)\right) \mathrm{d} s .
$$

Then, an inequality similar to (19) can be stated, namely

$$
\frac{v}{L_{x} L_{y} h} \limsup _{T \rightarrow \infty}\left\langle\|\mathbf{u}(s)\|^{2}\right\rangle_{T} \leq \frac{P}{L_{x}} \liminf _{T \rightarrow \infty} U_{T} .
$$

Hence, if we consider inequality 6.1 for the stationary statistical solution $\mu_{0}$ that extends the inferior limit of the time-averaged longitudinal bulk velocity of $\mathbf{u}(\mathbf{x}, t)$, we have

$$
\frac{v}{L_{x} L_{y} h} \limsup _{T \rightarrow \infty}\left\langle\|\mathbf{u}(s)\|^{2}\right\rangle_{T} \leq \frac{P}{L_{x}} \liminf _{T \rightarrow \infty} U_{T}=\frac{P U_{\mu_{0}}}{L_{x}} \leq \frac{\sqrt{3} h^{2}}{6 v} \frac{P^{2}}{L_{x}^{2}} .
$$


Similarly, considering inequality (53) for the same $\mu_{0}$ above, we have

$$
\begin{aligned}
P & \leq \frac{3 L_{x}}{2^{5 / 2} \pi^{2} h} U_{\mu_{0}}^{2}+\frac{27 L_{x} \pi v}{4 h^{2}} U_{\mu_{0}}+\frac{27 \pi^{2} L_{x} v^{2}}{2^{7 / 2} h^{3}} \\
& =\frac{3 L_{x}}{2^{5 / 2} \pi^{2} h}\left(\liminf _{T \rightarrow \infty} U_{T}\right)^{2}+\frac{27 L_{x} \pi v}{4 h^{2}}\left(\liminf _{T \rightarrow \infty} U_{T}\right)+\frac{27 \pi^{2} L_{x} v^{2}}{2^{7 / 2} h^{3}} .
\end{aligned}
$$

Substituting (73) into (72), we obtain

$$
\frac{v}{L_{x} L_{y} h} \limsup _{T \rightarrow \infty}\left\langle\|\mathbf{u}(s)\|^{2}\right\rangle_{T} \leq\left(\frac{3}{2^{5 / 2} \pi^{2} h}+\frac{27 \pi}{4} \frac{1}{R e}+\frac{27 \pi^{2}}{2^{7 / 2}} \frac{1}{R e^{2}}\right) \frac{\liminf _{T \rightarrow \infty} U_{T}^{3}}{h} .
$$

\section{The plane Poiseuille flow}

In order to analyze how sharp our estimates are, we calculate the characteristic quantities for a specific explicit flow. The wellknown explicit solution for the stationary version of the channel flow problem in this geometry is the plane Poiseuille flow:

$$
\mathbf{u}_{\text {Poiseuille }}(x, y, z)=\frac{P}{2 v L_{x}} z(h-z) \mathbf{e}_{1} .
$$

A straightforward calculation gives us the following estimates:

Proposition 8.1. We have, for the plane Poiseuille flow,

$$
\begin{aligned}
e_{\text {Poiseuille }} & =\frac{1}{2 L_{x} L_{y} h} \int_{\Omega} \frac{P^{2}}{4 v^{2} L_{x}^{2}} z^{2}(h-z)^{2} \mathrm{~d} x \mathrm{~d} y \mathrm{~d} z=\frac{h^{4}}{240 v^{2}}\left(\frac{P}{L_{x}}\right)^{2} ; \\
\epsilon_{\text {Poiseuille }} & =\frac{v}{L_{x} L_{y} h} \int_{\Omega} \frac{P^{2}}{4 v^{2} L_{x}^{2}}(h-2 z)^{2} \mathrm{~d} x \mathrm{~d} y \mathrm{~d} z=\frac{h^{2}}{12 v}\left(\frac{P}{L_{x}}\right)^{2} ; \\
U_{\text {Poiseuille }} & =\frac{h^{2}}{12 v} \frac{P}{L_{x}} ; \\
R e_{\text {Poiseuille }} & =\frac{h U_{\text {Poiseuille }}}{v}=\frac{h^{3}}{12 v^{2}} \frac{P}{L_{x}} ;
\end{aligned}
$$

and

$$
C_{f \text { Poiseuille }}=\frac{12}{R e_{\text {Poiseuille }}} .
$$

Remark 8.1. Notice that the upper bound estimates for the mean energy dissipation rate and mean longitudinal flow and the lower bound estimate for the skin-friction coefficient obtained in the previous sections are sharp since they are precisely those just presented for the plane Poiseuille flow. They are valid independently of the value of the applied pressure. As far as we know, these estimates were known only when the applied pressure is low, since only in this case is the plane Poiseuille flow globally asymptotically stable.

Remark 8.2. As remarked in [4], we can ensure the nonlinear stability of the plane Poiseuille flow from the positivity of the functional

$$
I_{\text {Poiseuille }}(\mathbf{v})=v\|\mathbf{v}\|^{2}+\int_{0}^{h} \int_{0}^{L_{y}} \int_{0}^{L_{x}} U_{1, \text { Poiseuille }}^{\prime}(z) v_{1}(\mathbf{x}) v_{3}(\mathbf{x}) \mathrm{d} x \mathrm{~d} y \mathrm{~d} z,
$$

where $U_{1, \text { Poiseuille }}=\left(P / 2 v L_{x}\right) z(h-z)$ is the velocity profile of the Poiseuille flow.

Note that this functional is very similar to the functional $H_{\mathbf{U}}$, and we can ensure its positivity by performing calculations very similar to the ones we did to ensure the positivity of $H_{\mathbf{U}}$. These calculations lead us to the following condition ensuring the positivity of $I_{\text {Poiseuille: }}$

$$
0<\frac{P}{L_{x}}<4 \sqrt{2} \pi^{2} \frac{v^{2}}{h^{3}} .
$$

Note that this upper bound condition is smaller than the upper bound condition stated in (53). Since for an applied pressure

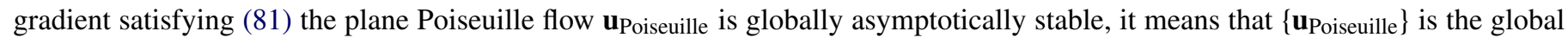


attractor for the Navier-Stokes equations in this case and, hence, there is a unique stationary statistical solution $\mu_{\text {Poiseuille }}$ which is a Dirac measure concentrated on $\left\{\mathbf{u}_{\text {Poiseuille }}\right.$. In this case, the estimates for the mean quantities are precisely those for the plane Poiseuille flow. In particular, this means that some of the estimates obtained in the previous sections are only needed for an applied pressure gradient larger than that given by condition (81).

\section{The rate of decrease of energy injection with respect to the scales of the flow}

In the classical theory of homogeneous turbulence it is argued that for turbulent flows, the energy injection is concentrated on the large scale motions, whereas the energy dissipated into heat due to the molecular viscosity occurs on scales that are much smaller.

In 1941, Kolmogorov [16] proposed that within a certain range of scales, much lower than the energy injection scales and greater than the energy dissipative scales, the energy is transferred to the small scales at a nearly constant rate equal to the energy dissipation rate. This mechanism is called the energy cascade, and sufficient conditions were rigorously derived in [9,12-14] for the existence of this phenomenon. This theory was proposed in the idealized case of locally homogeneous turbulence, away from the boundaries, with the injection of energy restricted to the large scales. However, it is well known from experiments that for wall bounded turbulence, this hypothesis needs to be corrected, see $[4,24,18]$. In particular, the energy injection occurs at arbitrarily small scales. The aim in this section is to give estimates for the rate of decrease of mean energy injection at progressively small scales.

By taking the scalar product of the Navier-Stokes equations with the component $\mathbf{u}_{\kappa^{\prime}, \kappa^{\prime \prime}}$ of the flow we find the energy equation for the scales of motion in the range $\left[\kappa^{\prime}, \kappa^{\prime \prime}\right)$. In the case $\kappa^{\prime \prime}<\infty$, we have the equality

$$
\frac{1}{2} \frac{\mathrm{d}}{\mathrm{d} t}\left|\mathbf{u}_{\kappa^{\prime}, \kappa^{\prime \prime}}\right|_{0}^{2}+v\left\|\mathbf{u}_{\kappa^{\prime}, \kappa^{\prime \prime}}\right\|^{2}+b\left(\mathbf{u}, \mathbf{u}, \mathbf{u}_{\kappa^{\prime}, \kappa^{\prime \prime}}\right)=\left(\left(\mathbf{f}_{P}\right)_{\kappa^{\prime}, \kappa^{\prime \prime}}, \mathbf{u}_{\kappa^{\prime}, \kappa^{\prime \prime}}\right) .
$$

In the case $\kappa^{\prime \prime}=\infty$, we have the inequality

$$
\frac{1}{2} \frac{\mathrm{d}}{\mathrm{d} t}\left|\mathbf{u}_{\kappa^{\prime}, \infty}\right|_{0}^{2}+v\left\|\mathbf{u}_{\kappa^{\prime}, \infty}\right\|^{2}+b\left(\mathbf{u}, \mathbf{u}, \mathbf{u}_{\kappa^{\prime}, \infty}\right) \leq\left(\left(\mathbf{f}_{P}\right)_{\kappa^{\prime}, \infty}, \mathbf{u}_{\kappa^{\prime}, \infty}\right) .
$$

The first term in the expressions above is the rate of change of kinetic energy; the second term represents the energy dissipation due to viscous effects; the third term represents the transfer of energy between different modes due to the convection term; and the last term represents the injection of energy due to the pressure gradient.

By dividing the equation by the volume $L_{x} L_{y} h$ of the periodic channel we find, in particular, that the energy injection rate per unit time and unit mass at each scale associated with a wavenumber $\kappa$ due to the pressure gradient is given by

$$
\mathfrak{F}_{\kappa}(\mathbf{u})=\frac{1}{L_{x} L_{y} h}\left(\left(\mathbf{f}_{P}\right)_{\kappa}, \mathbf{u}_{\kappa}\right) .
$$

The energy injection rate in a range of wavenumbers $\left[\kappa^{\prime}, \kappa^{\prime \prime}\right)$ is given by

$$
\mathfrak{F}_{\kappa^{\prime}, \kappa^{\prime \prime}}(\mathbf{u})=\frac{1}{L_{x} L_{y} h}\left(\left(\mathbf{f}_{P}\right)_{\kappa^{\prime}, \kappa^{\prime \prime}}, \mathbf{u}_{\kappa^{\prime}, \kappa^{\prime \prime}}\right) .
$$

The energy injection rate into the wavenumbers larger than or equal to a given wavenumber $\kappa$ is given by $\mathfrak{F}_{\kappa, \infty}(\mathbf{u})$.

The mean energy injection rate is given by the average value of those quantities with respect to a given stationary statistical solution. In order to estimate the mean energy injection rate at different length scales let us prove the following lemma.

Lemma 9.1. The forcing term component $\left(\mathbf{f}_{P}\right)_{\kappa}$, for a given wavenumber $\kappa$, is given by

$$
\left(\mathbf{f}_{P}\right)_{\kappa}= \begin{cases}\frac{2 L_{x}^{1 / 2} L_{y}^{1 / 2}}{h^{1 / 2}} \frac{P}{L_{x}} \frac{1}{\kappa} \mathbf{w}_{0,0, k}, & \text { if } \kappa=\frac{k \pi}{h}, k \in \mathbb{N}, k \text { odd } \\ 0 & \text { otherwise }\end{cases}
$$

Proof. First, notice that we can write

$$
\left(\mathbf{f}_{P}\right)_{\kappa}=\sum_{\kappa, l, k=\kappa}\left(\mathbf{f}_{P}, \mathbf{w}_{j, l, k}\right) \mathbf{w}_{j, l, k} .
$$

Each projection satisfies

$$
\left(\mathbf{f}_{P}, \mathbf{w}_{j, l, k}\right)=\frac{P}{L_{x}} \int_{\Omega} w_{j, l, k}^{1}(\mathbf{x}) \mathrm{d} \mathbf{x}
$$


where $w_{j, l, k}^{1}$ denotes the first component of the eigenvector $\mathbf{w}_{j, l, k}$. Now, by inspecting the expression (4) for $\mathbf{w}_{j, l, k}$, we notice that the integral (86) vanishes for all $(j, l) \neq(0,0)$. Thus, $(85)$ reduces to

$$
\left(\mathbf{f}_{P}\right)_{\kappa}=P L_{y} \sum_{\kappa_{0,0, k}=\kappa}\left(\int_{0}^{h} \hat{w}_{0,0, k}^{1}(z) \mathrm{d} z\right) \mathbf{w}_{0,0, k} .
$$

Furthermore, one can deduce from the Stokes problem and the expansion (4) that $\hat{\mathbf{w}}_{0,0, k}(z)$ satisfies the following onedimensional eigenvalue problem:

$$
\left\{\begin{array}{l}
-\frac{\partial^{2} \hat{w}_{0,0, k}^{1}(z)}{\partial z^{2}}=\lambda_{0,0, k} \hat{w}_{0,0, k}^{1}(z) \\
-\frac{\partial^{2} \hat{w}_{0,0, k}^{2}(z)}{\partial z^{2}}=\lambda_{0,0, k} \hat{w}_{0,0, k}^{2}(z) \\
\hat{w}_{0,0, k}^{2}(z)=0
\end{array}\right.
$$

with the normalized solution being

$$
\hat{\mathbf{w}}_{0,0, k}(0,0, z)=\frac{1}{L_{x}^{1 / 2} L_{y}^{1 / 2} h^{1 / 2}}\left(\sin \left(\frac{k \pi}{h} z\right), \sin \left(\frac{k \pi}{h} z\right), 0\right),
$$

and $\lambda_{0,0, k}=(k \pi / h)^{2}$. Then, we can once more reduce (85) to

$$
\left(\mathbf{f}_{P}\right)_{\kappa}= \begin{cases}P L_{y}\left(\int_{0}^{h} \hat{w}_{0,0, k}^{1}(z) \mathrm{d} z\right) \mathbf{w}_{0,0, k}, & \text { if } \kappa=\frac{k \pi}{h}, \text { for some } k \in \mathbb{N}, \\ 0, & \text { if } \kappa \neq \frac{k \pi}{h}, \text { for every } k \in \mathbb{N} .\end{cases}
$$

Then the result follows directly from the following calculation:

$$
\begin{aligned}
\int_{0}^{h} \hat{w}_{0,0, k}^{1}(z) \mathrm{d} z & =\frac{1}{L_{x}^{1 / 2} L_{y}^{1 / 2} h^{1 / 2}} \int_{0}^{h} \sin \left(\frac{k \pi}{h} z\right) \mathrm{d} z \\
& = \begin{cases}\left(\frac{2 h^{1 / 2}}{\pi L_{x}^{1 / 2} L_{y}^{1 / 2}}\right) \frac{1}{k}, & \text { if } k \text { is odd } \\
0, & \text { if } k \text { is even. }\end{cases}
\end{aligned}
$$

We now compute the energy injection rate at a given wavenumber $\kappa$.

Proposition 9.1. The energy injection rate at a given wavenumber $\kappa$ is given by

$$
\mathfrak{F}_{\kappa}(\mathbf{u})= \begin{cases}\frac{2}{L_{x}^{1 / 2} L_{y}^{1 / 2} h^{3 / 2}} \frac{P}{L_{x}} \frac{1}{\kappa} \hat{u}_{0,0, k}, & \text { if } \kappa=\frac{k \pi}{h}, k \in \mathbb{N}, k \text { odd }, \\ 0, & \text { otherwise. }\end{cases}
$$

Proof. We have, using Lemma 9.1,

$$
\begin{aligned}
\mathfrak{F}_{\kappa}(\mathbf{u}) \frac{1}{L_{x} L_{y} h}\left(\left(\mathbf{f}_{P}\right)_{\kappa}, \mathbf{u}_{\kappa}\right) & =\frac{2}{L_{x}^{1 / 2} L_{y}^{1 / 2} h^{3 / 2}} \frac{P}{L_{x}} \frac{1}{\kappa}\left(\mathbf{w}_{0,0, k}, \mathbf{u}_{\kappa}\right) \\
& =\frac{2}{L_{x}^{1 / 2} L_{y}^{1 / 2} h^{3 / 2}} \frac{P}{L_{x}} \frac{1}{\kappa} \hat{u}_{0,0, k},
\end{aligned}
$$

which completes the proof.

Proposition 9.2. The energy injection rate on the modes within the range $\left[\kappa^{\prime}, \kappa^{\prime \prime}\right), \kappa_{0} \leq \kappa^{\prime}<\kappa^{\prime \prime} \leq \infty$ is given by

$$
\mathfrak{F}_{\kappa^{\prime}, \kappa^{\prime \prime}}(\mathbf{u}) \frac{2}{L_{x}^{1 / 2} L_{y}^{1 / 2} h^{3 / 2}} \frac{P}{L_{x}} \sum_{\substack{\kappa^{\prime} \leq k \pi / h<\kappa^{\prime \prime} \\ \text { kodd }}} \frac{h}{k \pi} \hat{u}_{0,0, k} .
$$


Proof. We have

$$
\begin{aligned}
\mathfrak{F}_{\kappa^{\prime}, \kappa^{\prime \prime}}(\mathbf{u}) & =\frac{1}{L_{x} L_{y} h}\left(\mathbf{f}_{P}, \mathbf{u}_{\kappa^{\prime}, \kappa^{\prime \prime}}\right)=\frac{1}{L_{x} L_{y} h} \sum_{\kappa^{\prime} \leq \kappa_{j, l, k}<\kappa^{\prime \prime}}\left(\hat{\mathbf{f}_{P}}\right)_{j, l, k} \hat{u}_{j, l, k} \\
& =\frac{1}{L_{x} L_{y} h} \sum_{\substack{\kappa^{\prime} \leq \kappa_{0,0, k}<\kappa^{\prime \prime} \\
k \text { odd }}}\left(\hat{\mathbf{f}_{P}}\right)_{0,0, k} \hat{u}_{0,0, k}=\frac{2}{L_{x}^{1 / 2} L_{y}^{1 / 2} h^{3 / 2}} \frac{P}{L_{x}} \sum_{\substack{\kappa^{\prime} \leq k \pi / h<\kappa^{\prime \prime} \\
k \text { odd }}} \frac{h}{k \pi} \hat{u}_{0,0, k},
\end{aligned}
$$

which proves the proposition.

Finally, we present a more explicit estimate for the mean energy injection rate for modes larger than or equal to a given wavenumber.

Proposition 9.3. The mean energy injection rate on the modes larger than or equal to a given $\kappa \geq \kappa_{1}$ and with respect to an arbitrary stationary statistical solution satisfies

$$
\left\langle\mathfrak{F}_{\kappa, \infty}(\mathbf{u})\right\rangle \leq \frac{2}{3^{1 / 2} \nu^{1 / 2} h^{1 / 2}} \frac{P}{L_{x}} \frac{1}{\kappa^{3 / 2}} \epsilon^{1 / 2} .
$$

Proof. From the previous result we have

$$
\begin{aligned}
\mathfrak{F}_{\kappa, \infty}(\mathbf{u}) & =\frac{2}{L_{x}^{1 / 2} L_{y}^{1 / 2} h^{3 / 2}} \frac{P}{L_{x}} \sum_{\substack{k \pi / h \geq \kappa \\
k \text { odd }}} \frac{h}{k \pi} \hat{u}_{0,0, k} \\
& \leq \frac{2}{L_{x}^{1 / 2} L_{y}^{1 / 2} h^{3 / 2}} \frac{P}{L_{x}}\left(\sum_{\substack{k \pi / h \geq \kappa \\
k \text { odd }}} \frac{h^{4}}{k^{4} \pi^{4}}\right)^{1 / 2}\left(\sum_{\substack{k \pi / h \geq \kappa \\
\text { kodd }}} \frac{k^{2} \pi^{2}}{h^{2}} \hat{u}_{0,0, k}^{2}\right)^{1 / 2} \\
& \leq \frac{2}{L_{x}^{1 / 2} L_{y}^{1 / 2} h^{3 / 2}} \frac{P}{L_{x}}\left(\int_{\kappa}^{\infty} \frac{1}{\chi^{4}} \mathrm{~d} \chi\right)^{1 / 2}\left\|\mathbf{u}_{\kappa, \infty}\right\| .
\end{aligned}
$$

Thus,

$$
\begin{aligned}
\left\langle\mathfrak{F}_{\kappa, \infty}(\mathbf{u})\right\rangle & \leq \frac{2}{3^{1 / 2} L_{x}^{1 / 2} L_{y}^{1 / 2} h^{3 / 2}} \frac{P}{L_{x}} \frac{1}{\kappa^{3 / 2}}\left\langle\left\|\mathbf{u}_{\kappa, \infty}\right\|\right\rangle \\
& \leq \frac{2}{3^{1 / 2} L_{x}^{1 / 2} L_{y}^{1 / 2} h^{3 / 2}} \frac{P}{L_{x}} \frac{1}{\kappa^{3 / 2}}\left\langle\left\|\mathbf{u}_{\kappa, \infty}\right\|^{2}\right\rangle^{1 / 2} \\
& \leq \frac{2}{3^{1 / 2} v^{1 / 2} h^{1 / 2}} \frac{P}{L_{x}} \frac{1}{\kappa^{3 / 2}} \epsilon^{1 / 2},
\end{aligned}
$$

which completes the proof.

\section{Acknowledgments}

In the initial version of this article sent out for review, the lower bound on the skin friction given in Theorem 6.1 was $C_{f} \geq 2 \pi \sqrt{3} / \mathrm{Re}$. The improvement to the sharp (optimal) value given now was observed by us while the paper was under review and also independently by the referee. We are very grateful to the referee for his/her careful reading of the manuscript.

This work was partly supported by the National Science Foundation under the grant NSF-DMS-0604235, by the Research Fund of the Indiana University, by the Pronex in Turbulence, CNPq-FAPERJ, Brazil, grant E-26/171.198/2003, and by CNPq, Brasília, Brazil, grants 30.7953/2006-8 and 14.2011/2003-7.

\section{References}

[1] G.K. Batchelor, The Theory of Homogeneous Turbulence, Cambridge University Press, Cambridge, 1953.

[2] H. Bercovici, P. Constantin, C. Foias, O.P. Manley, Exponential decay of the power spectrum of turbulence, J. Statist. Phys. 80 (3-4) (1995) 579-602.

[3] P. Constantin, C.R. Doering, Variational bounds on energy dissipation in incompressible flows: Shear flow, Phys. Rev. E 49 (1994) $4087-4099$.

[4] P. Constantin, C.R. Doering, Variational bounds on energy dissipation in incompressible flows II: Channel flow, Phys. Rev. E 51 (1995) $3192-3198$.

[5] P. Constantin, C. Foias, Navier-Stokes Equation, University of Chicago Press, Chicago, 1989.

[6] C. Foias, Statistical study of the Navier-Stokes equations I, Rend. Sem. Mat. Univ. Padova 48 (1972) 219-348.

[7] C. Foias, Statistical study of the Navier-Stokes equations II, Rend. Sem. Mat. Univ. Padova 49 (1973) 9-123. 


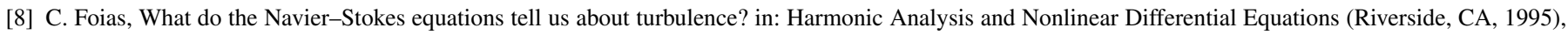
Contemp. Math. 208 (1997) 151-180.

[9] C. Foias, M.S. Jolly, O.P. Manley, Kraichnan turbulence via finite time averages, Commun. Math. Phys. 255 (2) (2005) $329-361$.

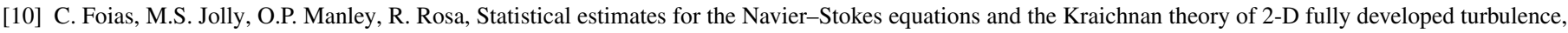
J. Statist. Phys. 108 (2002) 591-645.

[11] C. Foias, M.S. Jolly, O.P. Manley, R. Rosa, R. Temam, Kolmogorov theory via finite-time averages, Phys. D 212 (3-4) (2005) $245-270$.

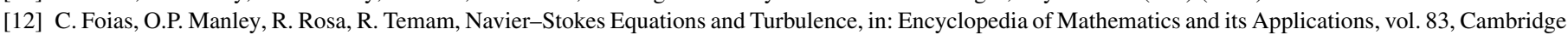
University Press, Cambridge, 2001.

[13] C. Foias, O.P. Manley, R. Rosa, R. Temam, Cascade of energy in turbulent flows, C.R. Acad. Sci. Paris, Série I 332 (2001) 509-514.

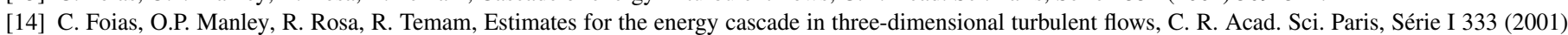
499-504.

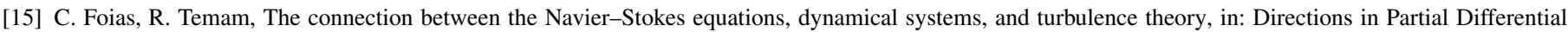
Equations, in: Publ. Math. Res. Center Univ. Wisconsin, 54, Academic Press, Boston, MA, 1987, pp. 55-73. Madison, WI, 1985.

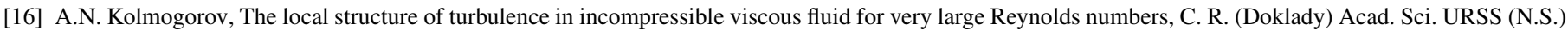
30 (1941) 301-305.

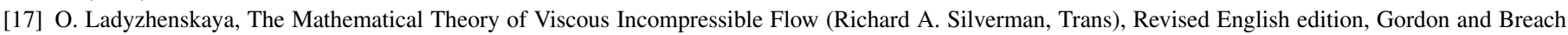
Science Publishers, New York, London, 1963.

[18] L. Prandtl, Über die ausgebildete Turbulenz, Z. Angew. Math. Mech. 5 (1925) 136-139.

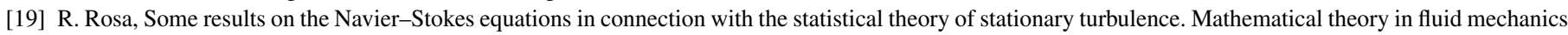
(Paseky, 2001), Appl. Math. 47 (6) (2002) 485-516.

[20] W. Rudin, Real and Complex Analysis, 3rd edition, McGraw-Hill Book Co, New York, 1987.

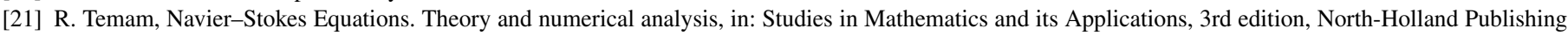
Co, Amsterdam-New York, 1984, Reedition in 2001 in the AMS Chelsea series, AMS, Providence.

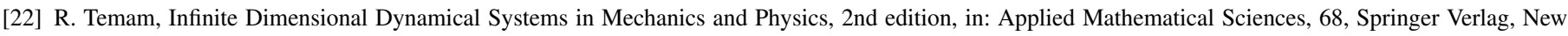
York, 1988.

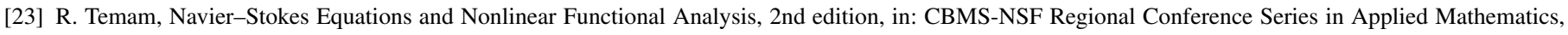
66, Society for Industrial and Applied Mathematics (SIAM), Philadelphia, PA, 1995.

[24] H. Tennekes, J.L. Lumley, A First Course in Turbulence, MIT, Cambridge, MA, 1972.

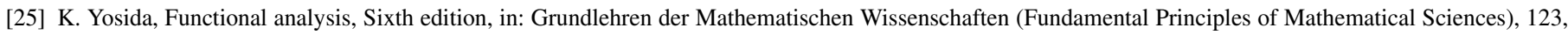
Springer-Verlag, Berlin-New York, 1980. 